\title{
LATTICE STRUCTURE OF WEYL GROUPS VIA REPRESENTATION THEORY OF PREPROJECTIVE ALGEBRAS
}

\author{
OSAMU IYAMA, NATHAN READING, IDUN REITEN, AND HUGH THOMAS
}

\begin{abstract}
This paper studies the combinatorics of lattice congruences of the weak order on a finite Weyl group $W$, using representation theory of the corresponding preprojective algebra $\Pi$. Natural bijections are constructed between important objects including join-irreducible congruences, join-irreducible (respectively, meet-irreducible) elements of $W$, indecomposable $\tau$-rigid (respectively, $\tau^{-}$-rigid) modules and layers of $\Pi$. The lattice-theoretically natural labeling of the Hasse quiver by join-irreducible elements of $W$ is shown to coincide with the algebraically natural labelling by layers of $\Pi$. We show that layers of $\Pi$ are nothing but bricks (or equivalently stones, or 2-spherical modules). The forcing order on join-irreducible elements of $W$ (arising from the study of lattice congruences) is described algebraically in terms of the doubleton extension order. We give a combinatorial description of indecomposable $\tau^{-}$-rigid modules for type $A$ and $D$.
\end{abstract}

\section{Contents}

1. Introduction

2. Preliminaries

2.1. Lattice-theoretic preliminaries

2.2. Weak order preliminaries

2.3. Algebraic preliminaries

2.4. Preliminaries on preprojective algebras

3. Homological characterizations of layers

4. Bijections: Theorems 1.1 and 1.3

5. Doubleton extension order on layer modules

6. Combinatorial description of indecomposable $\tau$-rigid modules

6.1. Type $A$

6.2. Type $D$

References

Key words and phrases. preprojective algebra, Weyl group, weak order, $\tau$-tilting theory, brick, join-irreducible element, lattice congruence.

2010 Mathematics Subject Classification. Primary 16G10, Secondary 06B10, 18E40, 20F55.

Osamu Iyama's work on this project was partially supported by JSPS Grant-in-Aid for Scientific Research (B) 24340004, (B) 16H03923, (C) 23540045 and (S) 15H05738. Nathan Reading's work on this project was partially supported by the National Science Foundation under grant numbers DMS-1101568 and DMS-1500949. Idun Reiten was supported by the FriNat grants 196600 and 231000 from the Research Council of Norway. Hugh Thomas's work on this project was partially supported by an NSERC Discovery Grant and the Canada Research Chairs program. The authors also gratefully acknowledge the hospitality of MSRI, Oberwolfach, Bielefeld University, and the Mittag-Leffler Institute. 


\section{INTRODUCTION}

Let $\Delta$ be a simply laced Dynkin diagram and $W$ the corresponding Weyl group. Once we fix an orientation $Q$ of $\Delta$, then the representation theory of $Q$ categorifies the root system associated with $\Delta$ in the sense that we have Gabriel's bijection between positive roots and indecomposable representations of $Q$. The preprojective algebra $\Pi$ of $\Delta$ unifies the representation theory of different quivers with the same underlying graph $\Delta$, and their various aspects has been studied, e.g. BKT, BGL, BIRS, CH, DR, GLS, Lu, KS, IN]. Mizuno [Mi] showed that the support $\tau$-tilting theory of $\Pi$ categorifies the Weyl group $W$ with the weak order in the following sense: There exists a bijection $W \ni w \mapsto I(w)$ from $W$ to the set s $\tau$-tilt $\Pi$ of support $\tau$-tilting $\Pi$-modules with the property that $v \leq w$ in the weak order on $W$ if and only if $I(v) \geq I(w)$ in the generation order on $\mathbf{s} \tau$-tilt $\Pi$. The ideal $I(w)$ was introduced in IR, BIRS and has been studied by several authors, e.g. AM, A, AIRT, BKT, GLS, K, Le, Ma, ORT, SY1. In what follows, we will overload the symbol $W$ to denote not only the group $W$, but also the weak order on $W$.

The weak order on $W$ is a lattice [BB]: a partial order such that meets (greatest lower bounds) and joins (least upper bounds) exist. It is enlightening to take a more algebraic point of view of lattices, viewing a lattice as a set with two binary operations (meet and join). Seen in this light, the categorification of $W$ by support $\tau$-tilting theory is the categorification of an algebraic object (a lattice) in terms of another algebraic object (a finite-dimensional algebra). In both of these algebraic settings, there is an important algebraic quotient operation. Quotients of the weak order are governed by lattice congruences, while quotients of the preprojective algebra are governed by ideals. A natural question is whether these two notions of quotient are related. The answer is yes, and the relationship turns out to be very nice.

This paper and a companion paper DIRRT concern the relationship between the two notions of quotient. In the other paper, we observe, for a more general algebra $A$ and an ideal $I$ of $A$, that $\mathrm{s} \tau$-tilt $(A / I)$ is a lattice quotient of $\mathrm{s} \tau$-tilt $A$ and give necessary conditions for lattice congruences which arise in this way from quotients of $\Pi$. We study the combinatorics of such algebraic quotients of the weak order in general, and in the special case where $\Pi / I$ is hereditary. We also work out, in detail, the combinatorics of algebraic quotients in type A.

Whereas DIRRT] starts with algebra quotients and determines what happens to the corresponding lattices, this paper starts from the other direction. Here, we start with the rich combinatorics of (arbitrary, not necessarily algebraic) lattice congruences of $W$ and find that it appears naturally within the representation theory of $\Pi$.

The set of all lattice congruences of $L$ form a lattice Con $L$, and the joinirreducible elements of Con $L$ are called the join-irreducible congruences (see Section 2.1 for details). The combinatorial approach to congruences of a finite lattice $L$ begins with the connection between arrows in the Hasse quiver of $L$, join-irreducible elements of $L$, and join-irreducible congruences on $L$. We will overload the symbol $W$, using it to denote the Hasse quiver of the weak order on $W$.

Our first main theorem connects join-irreducible elements of $W$ and join-irreducible congruences on $W$ to layers of $\Pi$. A $\Pi$-module is called a layer if it is isomorphic to $I(w) / I\left(w s_{i}\right)$ for an arrow $w s_{i} \rightarrow w$ in the Hasse quiver of $W$, see AIRT.

Theorem 1.1. There exist bijections between the following sets.

- The set j-Irr $W$ of join-irreducible elements of $W$.

- The set $\mathrm{m}$-Irr $W$ of meet-irreducible elements of $W$.

- The set $\mathrm{Con}_{\mathrm{JI}}(W)$ of join-irreducible congruences of $W$. 
- The set $\mathrm{i} \tau$-rigid $\Pi$ of indecomposable $\tau$-rigid $\Pi$-modules.

- The set $\mathrm{i} \tau^{-}$-rigid $\Pi$ of indecomposable $\tau^{-}$-rigid $\Pi$-modules.

- The set layer $\Pi$ of layers of $\Pi$.

We prove Theorem 1.1 and give explicit bijections as part of Theorem 4.1. The fact that join-irreducible elements, meet-irreducible elements, and join-irreducible congruences of $W$ are all in bijection is known, and this property of a lattice is called congruence uniformity (in the sense of Day [D]). This was proved in [CLM] (where in fact an equivalent property called boundedness was established).

The main content of Theorem 1.1 is the unexpectedly deep link between the representation theory of the preprojective algebra and the lattice theory of weak order on the corresponding Weyl group. As part of establishing this link, we have also proved some new results within the representation theory of preprojective algebras which we believe to be of independent interest. Our second main theorem gives two additional algebraic descriptions of layers of $\Pi$. We say that a $\Pi$-module $L$ is a brick if $\operatorname{End}_{\Pi}(L)$ is a division algebra, and a stone if $L$ is a brick satisfying $\operatorname{Ext}_{\Pi}^{1}(L, L)=0$ HHKU, KL. Let $\widehat{\Pi}$ be the preprojective algebra of the extended Dynkin type corresponding to $\Pi$. Let $\mathrm{D}^{\mathrm{b}}(\mathrm{fd} \widehat{\Pi})$ be the bounded derived category of finite dimensional $\widehat{\Pi}$-modules. An object $L \in \mathrm{D}^{\mathrm{b}}(\mathrm{fd} \widehat{\Pi})$ is called 2-spherical if $\operatorname{Hom}_{\mathrm{Db}^{\mathrm{b}}(\mathrm{fd} \widehat{\Pi})}(L, L[i])$ has dimension 1 for $i=0,2$ and 0 otherwise. (See $[\mathrm{ST}]$.)

Theorem 1.2. The following classes of $\Pi$-modules are the same.

- Layers of $\Pi$.

- Bricks of $\Pi$.

- Stones of $\Pi$.

- П-modules which are 2-spherical as $\widehat{\Pi}$-modules,

An observation related to Theorem[1.2 was given by Bolten Bol] and Sekiya and Yamaura SY2.

Our third main result concerns the interplay between arrows in the Hasse quiver of $W$, join-irreducible elements of $W$, and join-irreducible congruences on $W$. We refer to Section 2.1 for details about the following notions. Given any arrow $x \rightarrow y$ in the Hasse quiver of an arbitrary finite lattice $L$, define $\operatorname{con}(x, y)$ to be the smallest congruence on $L$ such that $x \equiv y$. This is a join-irreducible congruence. If $j$ is a join-irreducible element of $L$, we write $j_{*}$ for the unique element covered by $j$ in $L$. The congruence $\operatorname{con}\left(j, j_{*}\right)$ is thus join-irreducible, and it turns out that every join-irreducible element is $\operatorname{con}\left(j, j_{*}\right)$ for some $j$. When $L$ is the weak order on $W$, the map $j \mapsto \operatorname{con}\left(j, j_{*}\right)$ is the bijection from join-irreducible elements of $W$ to joinirreducible congruences from Theorem 1.1. Since each Hasse arrow of $W$ specifies a join-irreducible congruence, and since join-irreducible congruences are in bijection with join-irreducible elements, we obtain a labelling of the Hasse arrows of $W$ by join-irreducible elements. We call this the join-irreducible labelling of $W$. Besides this labelling coming from lattice theory, there is a labelling of the Hasse quiver coming from representation theory, namely the layer labelling. This labels a Hasse arrow $w s_{i} \rightarrow w$ by the layer $I(w) / I\left(w s_{i}\right)$. The layer labellings for type $A_{2}$ and $A_{3}$ are given in Figures 1 and 2 .

Theorem 1.3. The map $j \mapsto I\left(j_{*}\right) / I(j)$ takes the join-irreducible labelling of $W$ to the layer labelling on $W$. That is, given a Hasse arrow $w s_{i} \rightarrow w$ labelled by the join-irreducible element $j$, which covers the element $j_{*}$, the layer label on $w s_{i} \rightarrow w$ is $I\left(j_{*}\right) / I(j)$.

This is also proved as part of Theorem 4.1, which gives a commutative diagram shown in Figure 3 between important objects. We include this diagram here, although some elements of it have not yet been explained, as a road map to the major 


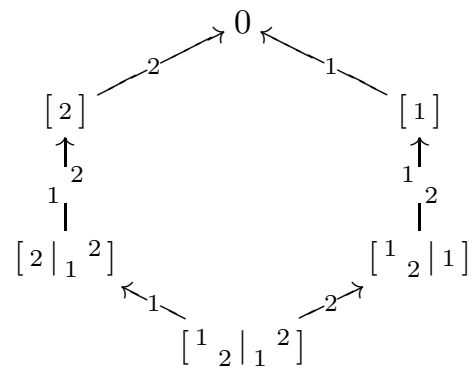

FiguRE 1. Layer labelling for $A_{2}$

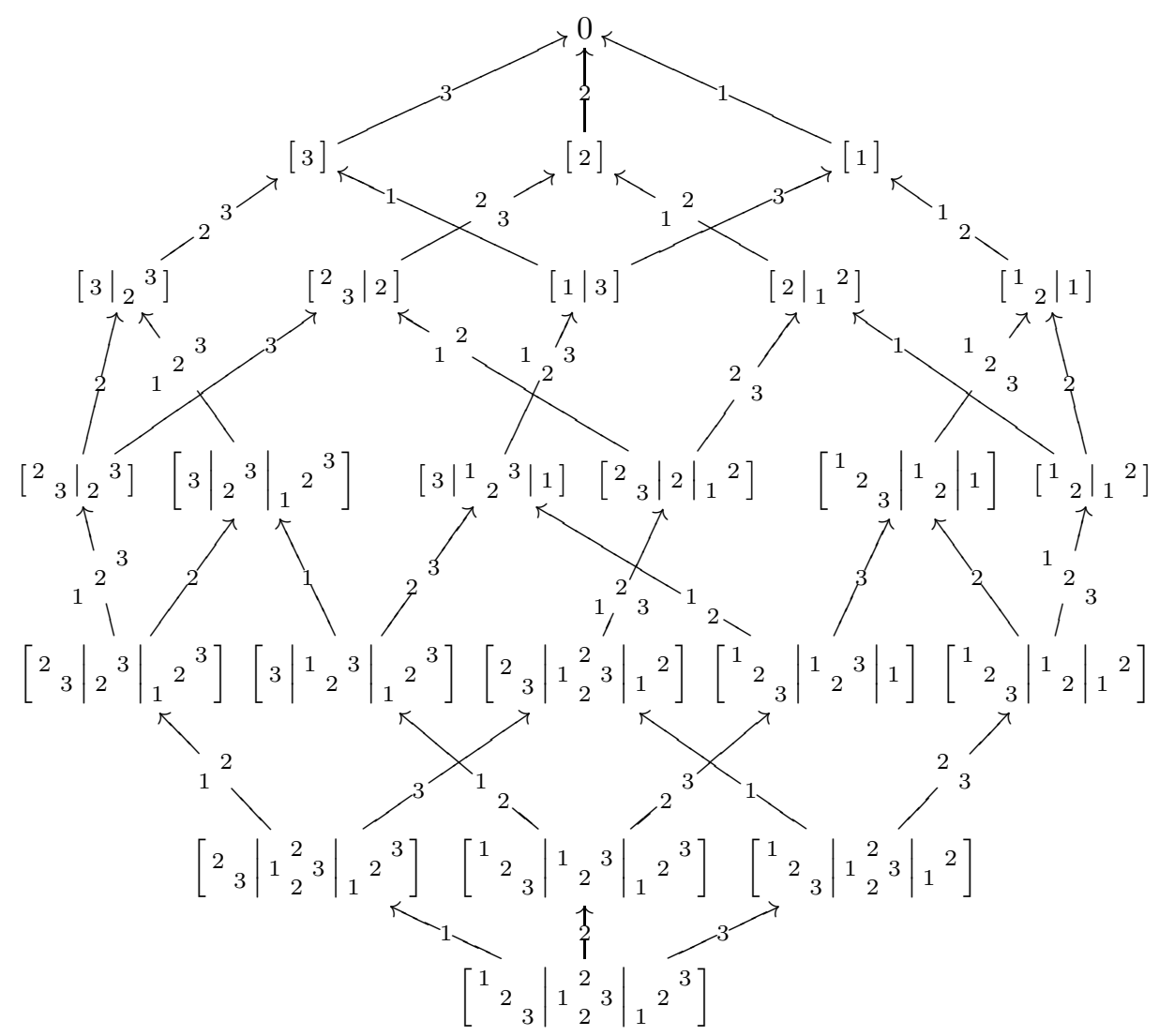

Figure 2. Layer labelling for $A_{3}$

results of the paper. The maps are bijections or surjections as marked with tildes " $\sim$ " or double-headed arrows.

Given two Hasse arrows $x \rightarrow y$ and $x^{\prime} \rightarrow y^{\prime}$ of a lattice $L$, we say that $x \rightarrow y$ forces $x^{\prime} \rightarrow y^{\prime}$ if $\operatorname{con}(x, y) \geq \operatorname{con}\left(x^{\prime}, y^{\prime}\right)$ in $\operatorname{Con}(L)$. In other words, $x \rightarrow y$ forces $x^{\prime} \rightarrow y^{\prime}$ if every congruence setting $x \equiv y$ also sets $x^{\prime} \equiv y^{\prime}$. In the weak order on $W$, the forcing order on Hasse arrows restricts to a partial order on Hasse arrows of the form $j \rightarrow j_{*}$ such that $j$ is join-irreducible. We think of this as a partial order on join-irreducible elements and call it the forcing order on join-irreducible elements of $W$, 


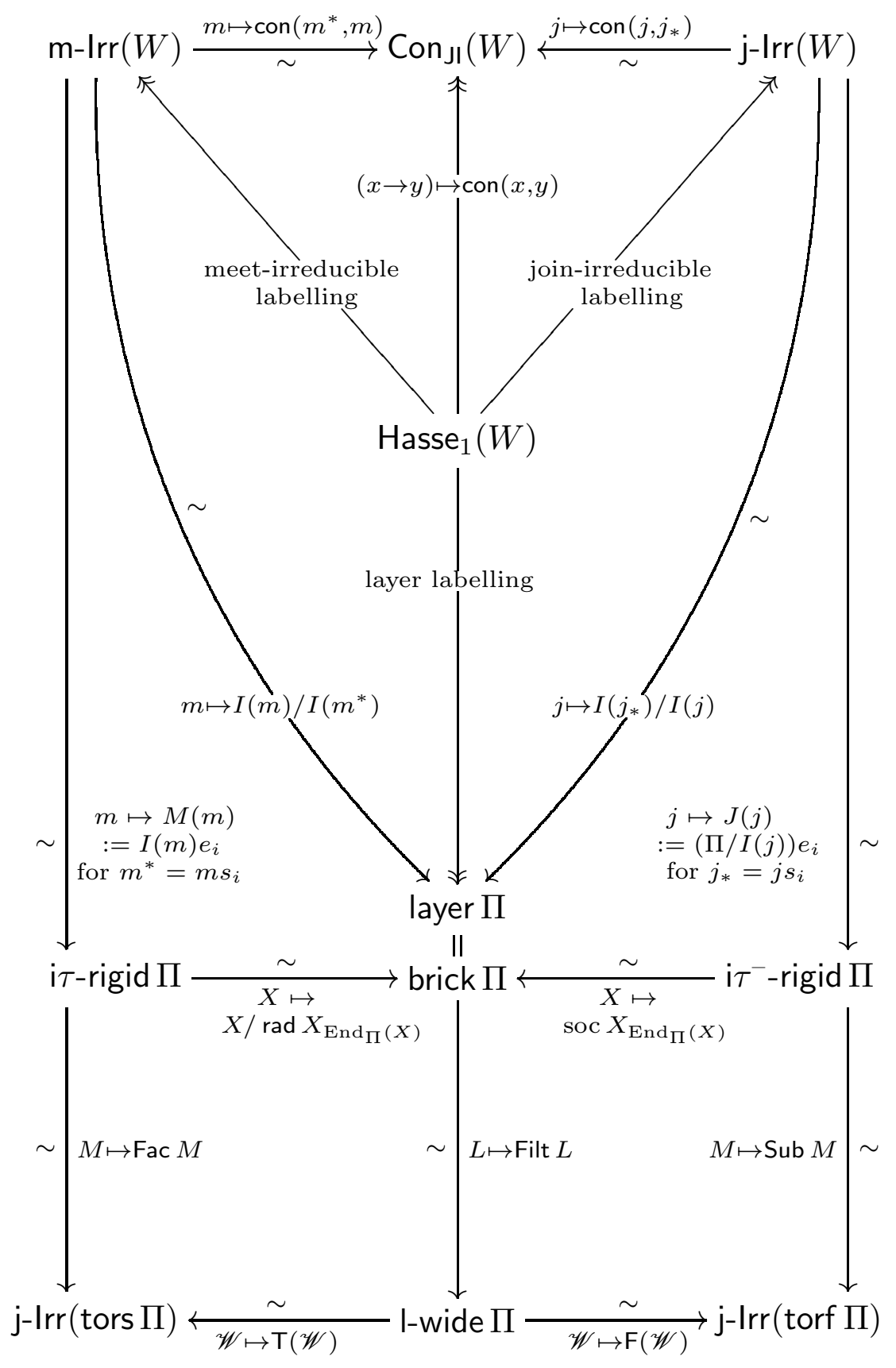

Figure 3. The correspondences established in Theorem 4.1

We say that a pair of layer modules $X, Y$ form a doubleton if $\operatorname{Ext}_{\Pi}^{1}(Y, X)$ and $\operatorname{Ext}_{\Pi}^{1}(X, Y)$ are one-dimensional, and the corresponding extensions are again layer modules. We define the doubleton extension order on layer modules to be the transitive closure of the relation with $A>B$ if there exists a doubleton $A, C$ such that $B$ is the extension of $A$ by $C$ or of $C$ by $A$. Our fourth main result is the following, 
Theorem 1.4. The map $j \mapsto I\left(j_{*}\right) / I(j)$ is an isomorphism from the forcing order on join-irreducible elements of $W$ to the doubleton extension order on layer modules of $\Pi$.

In addition to these general results, we show that in type $A_{n}$, the doubleton extension order coincides with the reverse of the subfactor order (see Theorem 5.5). Furthermore, we give an explicit combinatorial description of the indecomposable $\tau$-rigid modules in types $A_{n}$ and $D_{n}$ in terms of the Young diagrams associated with the join-irreducible elements in the Weyl group $W$ (see Theorems 6.1, 6.5 and 6.12).

\section{Preliminaries}

In this section, we review the necessary background on lattices, the weak order, and finite-dimensional algebras.

2.1. Lattice-theoretic preliminaries. Proofs and additional details for the material reviewed here can be found in [R2, Sections 9-5 and 9-6].

For any poset $P$, we say that $x$ covers $y$, and we write $x>y$, if $x>y$, and there is no $z \in P$ such that $x>z>y$. We represent $P$ by its Hasse quiver Hasse $(P)=\left(P\right.$, Hasse $\left._{1}(P)\right)$, whose vertex set is $P$, and whose arrow set $\operatorname{Hasse}_{1}(P)$ consists of all arrows $v \rightarrow w$ where $v$ covers $w$.

Given a subset $S$ of $P$, if there is a unique smallest element which is greater than or equal to all elements in $S$, then this least upper bound is called the join of $S$ and denoted $\bigvee S$. Similarly, if there is a unique largest element in $P$ that is less than or equal to all elements in $S$, then this element is called the meet of $S$ and denoted $\bigwedge S$. A lattice $L$ is a poset in which every pair $a, b$ of elements in $L$ have both a meet $a \wedge b$ and a join $a \vee b$, and a complete lattice $L$ is a poset in which every subset $S$ of $L$ has both a meet and a join. (Every finite subset of a lattice $L$ has both a meet and a join, but an infinite lattice fails to be complete if it has some infinite subset without a meet or without a join.)

We restrict our attention to finite lattices in this paper. Some of the assertions made here for finite lattices hold for infinite lattice as well, but some do not.

An element $j$ of a finite lattice $L$ is called join-irreducible, whenever $j=a \vee b$ for some $a, b \in L$, either $a=j$ or $b=j$ or both, and $j$ is not the minimum element of $L$. Equivalently, $j$ is join-irreducible if and only if it covers exactly one element of $L$. We write $j_{*}$ for the unique element covered by a join-irreducible element $j$. Dually, a meet-irreducible element of $L$ is an element $m$ that is covered by a unique element $m^{*}$. The set of join-irreducible (respectively, meet-irreducible) elements of $L$ is denoted j-Irr $L$ (respectively, $\mathrm{m}$-Irr $L$ ).

A (lattice) congruence on a lattice $L$ is an equivalence relation $\Theta$ having the property that the $\Theta$-class of $a \vee b$ depends only on the $\Theta$-class of $a$ and the $\Theta$-class of $b$, and having the same property for meets. Given a congruence $\Theta$ on $L$, the set $L / \Theta$ of $\Theta$-classes has a well-defined meet and join operation, making $L / \Theta$ a lattice called the quotient of $L$ modulo $\Theta$.

The set of all equivalence relations on a given set $L$ forms a lattice, where the meet of two relations is given by the intersection of relations and the join of two relations is given by the transitive closure of union of relations. When $L$ is a lattice, the set $\operatorname{Con}(L)$ consisting of congruences of $L$ is a sublattice of the lattice of equivalence relations. Furthermore, $\operatorname{Con}(L)$ is a distributive lattice. We denote by $\operatorname{Con}_{\mathrm{JI}}(L)$ the set of all join-irreducible congruences. As mentioned in the introduction, we have a surjective map Hasse $_{1}(L) \rightarrow$ Con $_{\mathrm{JI}}(L)$ sending an arrow $x \rightarrow y$ to $\operatorname{con}(x, y)$. Here $\operatorname{con}(x, y)$ is the meet, in Con $(L)$, of all congruences with $x \equiv y$. A congruence $\Theta$ on a finite lattice $L$ is determined completely by the set of cover relations $x>y$ 
in $L$ such that $x \equiv y$ modulo $\Theta$. It is also determined uniquely by the set of join-irreducible elements $j$ in $L$ such that $j \equiv j_{*}$ modulo $\Theta$, and thus we have an injective map Con $(L) \rightarrow 2^{\text {j-Irr }(L)}$.

The map from cover relations $x>y$ to join-irreducible congruences is typically not one-to-one. The restriction of the map to cover relations of the form $j \rightarrow j_{*}$ is also surjective onto join-irreducible congruences, but may still fail to be one-to-one. A lattice is called congruence uniform if the map $j \mapsto \operatorname{con}\left(j, j_{*}\right)$ is injective (and thus a bijection) from join-irreducible elements to join-irreducible congruences and the map $m \rightarrow \operatorname{con}\left(m^{*}, m\right)$ is injective (and thus a bijection) from meet-irreducible elements to join-irreducible congruences. A finite congruence uniform lattice is always semidistributive. This means that if $x \vee y=x \vee z$ then $x \vee(y \wedge z)=x \vee y$ and if $x \wedge y=x \wedge z$ then $x \wedge(y \vee z)=x \wedge y$.

Since $\operatorname{Con}(L)$ is a finite distributive lattice, the Fundamental Theorem of Finite Distributive Lattices says that its elements are naturally identified with order ideals in the subposet $\operatorname{Con}_{\mathrm{JI}}(L)$ of $\operatorname{Con}(L)$. When $L$ is congruence uniform, the subposet Con $_{\mathrm{JI}}(L)$ induces a partial order on the join-irreducible elements of $L$, which we call the forcing order. A congruence $\Theta \in \operatorname{Con}(L)$ corresponds to the order ideal in $\operatorname{Con}_{\mathrm{Jl}}(L)$ consisting of those join-irreducible congruences below $\Theta$ in $\operatorname{Con}(L)$ (i.e. finer than $\Theta$ as equivalence relations). These are the join-irreducible congruences $\operatorname{con}\left(j, j_{*}\right)$ such that $j \equiv j_{*}$ modulo $\Theta$. The forcing order on join-irreducible elements sets $j \leq j^{\prime}$ if and only if $j \equiv j_{*}$ modulo $\operatorname{con}\left(j^{\prime}, j_{*}^{\prime}\right)$.

As mentioned above, each cover relation $x>y$ in a finite lattice defines a join-irreducible congruence of $L$. In a finite congruence uniform lattice $L$, each join-irreducible congruence is $\operatorname{con}\left(j, j_{*}\right)$ for a unique join-irreducible element $j$ of $L$. The map $\mathrm{Hasse}_{1}(L) \rightarrow \mathrm{j}$-Irr $(L)$ sending the arrow $x \rightarrow y$ to the unique $j$ with $\operatorname{con}\left(j, j_{*}\right)=\operatorname{con}(x, y)$ is called the join-irreducible labelling of $L$. Each joinirreducible congruence is also $\operatorname{con}\left(m^{*}, m\right)$ for a unique meet-irreducible element $m$, and the map $\operatorname{Hasse}_{1}(L) \rightarrow \mathrm{m}-\operatorname{Irr}(L)$ sending $x \rightarrow y$ to the unique $m$ with $\operatorname{con}\left(m^{*}, m\right)=\operatorname{con}(x, y)$ is called the meet-irreducible labelling of $L$. These labellings are described explicitly as follows.

The following proposition is $[\mathrm{R} 2$, Proposition 9-5.20]. Since that proposition's proof is left to an exercise, we give a proof here.

Proposition 2.1. Let $L$ be a finite congruence uniform lattice and let $x \rightarrow y$ be an arrow in $\operatorname{Hasse}(L)$.

(a) The join-irreducible label on $x \rightarrow y$ is $j=\bigwedge\{z \in L: z \leq x, z \not \leq y\}$. Furthermore, $j \leq x$ but $j \not \leq y$.

(b) The meet-irreducible label on $x \rightarrow y$ is $m=\bigvee\{z \in L: z \geq y, z \nsupseteq x\}$. Furthermore, $m \geq y$ but $m \geq x$.

In particular, if $j$ is a join-irreducible element and $m$ is a meet-irreducible element with $\operatorname{con}\left(j, j_{*}\right)=\operatorname{con}\left(m^{*}, m\right)$, then $j=\bigwedge\left\{z \in L: z \leq m^{*}, z \not \leq m\right\}$ and $m=\bigvee\left\{z \in L: z \geq j_{*}, z \nsupseteq j\right\}$.

Proof. The last statements are special cases of assertions (a) and (b). Assertions (a) and (b) are dual to each other, so by symmetry it is enough to prove (a). To do so, it is enough to show that $j$ is join-irreducible and that $\operatorname{con}\left(j, j_{*}\right)=\operatorname{con}(x, y)$.

Recall that a congruence uniform finite lattice is also semidistributive. Every element $z$ of $\{z \in L: z \leq x, z \not \leq y\}$ has $z \vee y=x$, so applying semidistributivity several times, we see that $j \vee y=x$, so in particular $j \not \subset y$. It is immediate that $j \leq x$. If $j$ covers elements $a$ and $b$, then $a \leq y$ and $b \leq y$. But if $a \neq b$, then $j$ is a minimal upper bound for $a$ and $b$, so it must equal $a \vee b$. Since $y$ is another upper bound for $a$ and $b$, we reach the contradiction $j \leq y$. We conclude that $j$ covers 


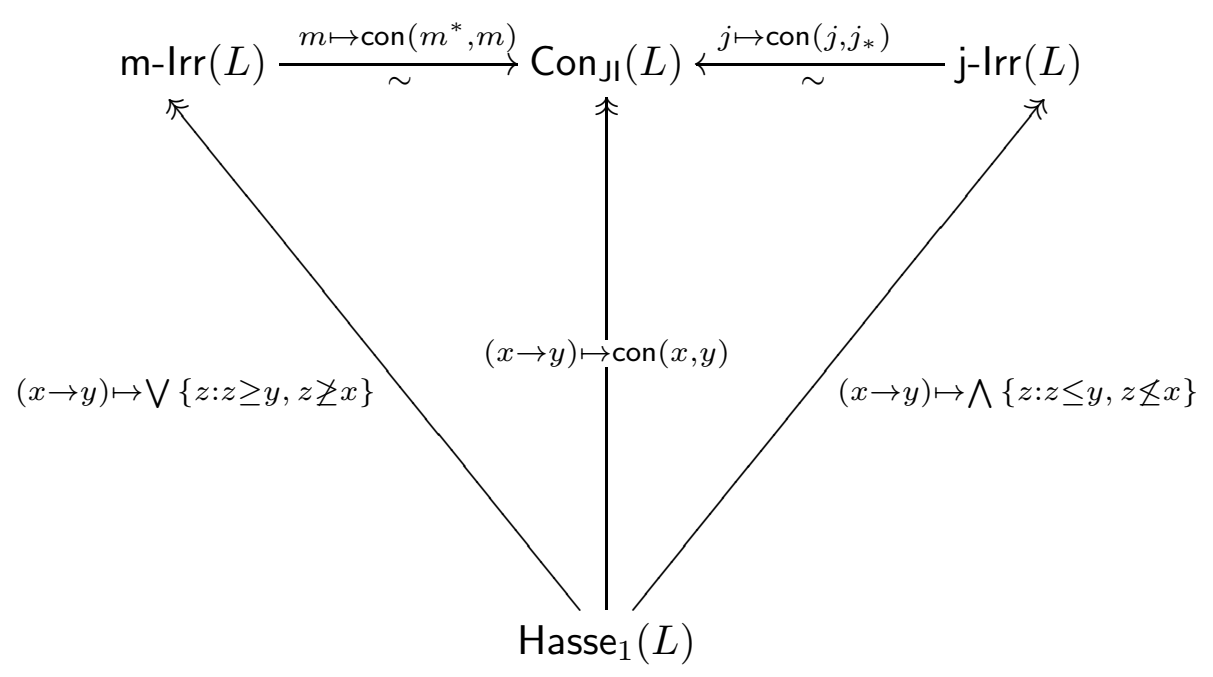

FIGURE 4. Join-irreducible elements, meet-irreducible elements, and congruences in a finite congruence uniform lattice

at most one element. If $j$ covers no element, then $j$ is the minimal element of $L$, contradicting again the fact that $j \not \leq y$. We see that $j$ is join-irreducible.

We saw that $j \vee y=x$ and we also verify easily that $j \wedge y=j_{*}$. If $\Theta$ is a congruence with $j \equiv j_{*}$, then $j \vee y \equiv j_{*} \vee y$, or in other words $x \equiv y$. Conversely, if $\Theta$ has $x \equiv y$, then $j \wedge x \equiv j \wedge y$, or in other words $j \equiv j_{*}$. We see that $\Theta$ has $j \equiv j_{*}$ if and only if $x \equiv y$, so that $\operatorname{con}\left(j, j_{*}\right)=\operatorname{con}(x, y)$.

We summarize some of what we know about join-irreducible elements, meetirreducible elements, and congruences in a finite congruence uniform lattice in Figure 4. If $L$ is a finite congruence uniform lattice with Hasse quiver Hasse $(L)$, joinirreducible elements j-Irr $(L)$, meet-irreducible elements $m-\operatorname{Irr}(L)$, and join-irreducible congruences Con $_{\mathrm{JI}}(L)$, then the diagram in Figure 4 commutes and the maps are bijections or surjections as marked with tildes " " or double-headed arrows.

Let $x$ be an element of a finite lattice $L$. The expression $x=\bigvee S$ is the canonical join representation of $x$ if no proper subset of $S$ joins to $x$ and if every joinrepresentation $x=\bigvee T$ has the property that for all $s \in S$, there exists $t \in$ $T$ with $s \leq t$. An element $x$ may fail to have a canonical join representation, but the canonical join representation of $x$ is unique if it exists. The canonical meet representation is defined dually. The semidistributive property of a finite lattice $L$, described above, is equivalent to the property that every element of $L$ has a canonical join representation and a canonical meet representation. A finite congruence uniform lattice is in particular semidistributive, and canonical join and meet representations can be described in terms of the join-irreducible labelling and meet-irreducible labelling as follows.

The following proposition is [ $\mathrm{R} 2$, Proposition 9-5.30]. Since that proposition's proof is also left to an exercise, we give a proof here.

Proposition 2.2. If $L$ is a finite congruence uniform lattice, then the canonical join representation of $x \in L$ is $x=\bigvee J$, where $J$ is the set of join-irreducible labels on arrows starting at $x$ in Hasse $(L)$. The canonical meet representation is $x=\bigwedge M$, where $M$ is the set of meet-irreducible labels on arrows ending at $x$ in $\operatorname{Hasse}(L)$. 
Proof. We prove the statement for canonical join representations, using Proposition 2.1 throughout. The other statement is dual.

First, we check that $x=\bigvee J$. On the one hand, each $j \in J$ is below $x$ by Proposition 2.1, so $\bigvee J \leq x$. If $\bigvee J<x$, then there exists $y$ with $x>y \geq \bigvee J$. But the label of $x \rightarrow y$ is in $J$ and is not below $y$, which is a contradiction.

Next, we show that no proper subset of $J$ joins to $x$. If $x \rightarrow y$ and $J^{\prime}=$ $J \backslash\{\bigwedge\{z \in L: z \leq x, z \not \leq y\}\}$, then for any other $y^{\prime}$ with $x \rightarrow y^{\prime}$, we have $y \leq x$ by $y \nsupseteq y^{\prime}$, so $y \geq \bigwedge\left\{z \in L: z \leq x, z \not \leq y^{\prime}\right\}$. Therefore every element of $J^{\prime}$ is $\leq y$, so $\bigvee J^{\prime}<x$.

Finally, we show that, if $x=\bigvee T$ for some $T \subseteq L$, then for all $j \in J$, there exists $t \in T$ with $j \leq t$. Let $y$ have $x \rightarrow y$ and $j=\bigwedge\{z \in L: z \leq x, z \not \leq y\}$. Every element of $T$ is $\leq x$, and if every element of $T$ is $\leq y$, then $\bigvee T \leq y$, contradicting the supposition that $x=\bigvee T$. Thus there exists some element $t$ of $T$ with $t \leq x$ and $t \not \leq y$, and this element is above $j$ by definition.

A polygon in a finite lattice $L$ is an interval $[x, y]$ such that $\{z \in L: x<z<y\}$ consists of two disjoint nonempty chains. (Thus the Hasse quiver of $[x, y]$ is a cycle with one source and one sink.) The lattice $L$ is polygonal if the following two conditions hold: First, if distinct elements $y_{1}$ and $y_{2}$ both cover an element $x$, then $\left[x, y_{1} \vee y_{2}\right]$ is a polygon; and second, if an element $y$ covers distinct elements $x_{1}$ and $x_{2}$, then $\left[x_{1} \wedge x_{2}, y\right]$ is a polygon.

If $L$ is a polygonal lattice, then we define a quiver $\operatorname{FPoly}(L)$ whose set of vertices is Hasse $_{1}(L)$, with arrows defined in every polygon $P$ of $L$ as follows. The two arrows into the bottom element of $P$ are called bottom arrows, while the two arrows from the top element of $P$ are called top arrows, and all other arrows of $P$ are called side arrows. Every bottom arrow of $P$ has an arrow (in FPoly $(L)$ ) to the opposite top arrow in $P$ (i.e. the top arrow in the opposite chain) and has an arrow to every side arrow in $P$. Every top arrow of $P$ has an arrow to the opposite bottom arrow and every side arrow in $P$. For example, a square and hexagon in $L$ would contribute to $\operatorname{FPoly}(L)$ as indicated below.
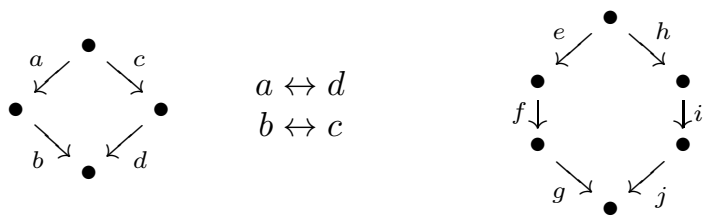

$$
\begin{array}{ccc}
e \leftrightarrow j & e \rightarrow f & e \rightarrow j \\
g \leftrightarrow h & g \rightarrow f & g \rightarrow i \\
& h \rightarrow f & h \rightarrow i \\
& j \rightarrow f & j \rightarrow i
\end{array}
$$

The following is [R2, Theorem 9-6.5].

Theorem 2.3. If $L$ is a finite polygonal lattice, and $x \rightarrow y$ and $x^{\prime} \rightarrow y^{\prime}$ are arrows in Hasse $(L)$, then $\operatorname{con}\left(x^{\prime}, y^{\prime}\right) \leq \operatorname{con}(x, y)$ if and only if there is a directed path from $x \rightarrow y$ to $x^{\prime} \rightarrow y^{\prime}$ in FPoly $(L)$.

When $L$ is also congruence uniform, the quiver $\operatorname{FPoly}(L)$ is closely related to the forcing order on join-irreducible elements of $L$, as described in the following corollary, which is immediate by combining Theorem 2.3 with facts about finite congruence uniform lattices already given in this section. We say that a quiver $Q$ is strongly connected if given any two vertices $x$ and $y$ of the quiver, there exists a path from $x$ to $y$ and a path from $y$ to $x$. A strongly connected component of $Q$ is a set of vertices of $Q$ that is maximal with respect to inducing a strongly connected subquiver.

Corollary 2.4. Suppose $L$ is a finite, polygonal, congruence uniform lattice. Then each strongly connected component of FPoly $(L)$ contains exactly one arrow in $\operatorname{Hasse}(L)$ of the form $j \rightarrow j_{*}$ where $j$ is join-irreducible. This bijection between join-irreducible 
elements of $L$ and strongly connected components of $\mathrm{FPoly}(L)$ is an isomorphism from the forcing order on join-irreducible elements of $L$ to the partial order induced by $\mathrm{FPoly}(L)$ on its strongly connected components.

Informally, the corollary says that all forcing in a finite polygonal, congruence uniform lattice comes from forcing in polygons. Indeed, the notation FPoly $(L)$ suggests the phrase "forcing in polygons."

We define another quiver SFPoly $(L)$, again with the set of vertices given by Hasse $_{1}(L)$, but with strictly fewer arrows than FPoly $(L)$. The notation SFPoly $(L)$ suggests the phrase "strong forcing in polygons." In SFPoly $(L)$, every bottom arrow of a polygon $P$ has an arrow only to the opposite top arrow in $P$ and every top arrow of $P$ has an arrow only to the opposite bottom arrow in $P$. Thus from the square and hexagon as labelled above, SFPoly $(L)$ gets arrows as indicated below.

$$
a \leftrightarrow d \quad b \leftrightarrow c \quad e \leftrightarrow j \quad g \leftrightarrow h
$$

Strong forcing in polygons controls whether two Hasse arrows in a finite polygonal, congruence uniform lattice determine the same congruence, as described in the following corollary.

Corollary 2.5. Suppose $L$ is a finite polygonal, congruence uniform lattice and let $x \rightarrow y$ and $x^{\prime} \rightarrow y^{\prime}$ be arrows of Hasse $(L)$. Then $\operatorname{con}(x, y)=\operatorname{con}\left(x^{\prime}, y^{\prime}\right)$ if and only if there is a directed path (or equivalently a path) in $\operatorname{SFPoly}(L)$ from $x \rightarrow y$ to $x^{\prime} \rightarrow y^{\prime}$.

Proof. The "if" direction is immediate by Theorem 2.3, the fact that every arrow is $\operatorname{SFPoly}(L)$ is also an arrow in $\operatorname{FPoly}(L)$, and the fact that arrows in SFPoly $(L)$ come in opposite pairs. We prove the converse by proving that for every Hasse arrow $x \rightarrow y$, there is a directed path in $\operatorname{SFPoly}(L)$ from $x \rightarrow y$ to $j \rightarrow j_{*}$, where $j$ is the unique join-irreducible element with $\operatorname{con}\left(j, j_{*}\right)=\operatorname{con}(x, y)$. Proposition 2.1 says in particular that $x \geq j$, so we can argue by induction on the length of a longest maximal chain from $j$ to $x$. Choose $z$ with $x>z \geq j$ and set $y^{\prime}=y \wedge z$. Since $L$ is polygonal, $\left[y^{\prime}, x\right]$ is a polygon $P$, and $x \rightarrow y$ is a top arrow in $P$. Choosing $x^{\prime}$ so that $x^{\prime} \rightarrow y^{\prime}$ is the bottom arrow opposite $x \rightarrow y$ (i.e. in the other chain of $P$ ), we have $\operatorname{con}\left(x^{\prime} \rightarrow y^{\prime}\right)=\operatorname{con}(x \rightarrow y)$ by Theorem 2.3. Thus $\operatorname{con}\left(j, j_{*}\right)=\operatorname{con}\left(x^{\prime}, y^{\prime}\right)$, and thus $x^{\prime} \geq j$. By induction, there is a directed path in $\operatorname{SFPoly}(L)$ from $x^{\prime} \rightarrow y^{\prime}$ to $j \rightarrow j_{*}$, and using an arrow in $P$, we obtain a directed path in $\operatorname{SFPoly}(L)$ from $x \rightarrow y$ to $j \rightarrow j_{*}$.

2.2. Weak order preliminaries. Fix a simply-laced Dynkin type (i.e., one of $A_{n}$ for $n \geq 1, D_{n}$ for $n \geq 4, E_{6}, E_{7}$, or $\left.E_{8}\right)$. Let $W$ be the finite Weyl group of that type. For background on Weyl groups, see $\mathrm{BB}$.

Let $S=\left\{s_{1}, \ldots, s_{n}\right\}$ be the set of simple reflections of $W$. By definition, any element $w$ of $W$ can be written as a product of the simple reflections. Such an expression for $w$ of minimal length is called reduced. The length of a reduced expression is the length of $w$, denoted $\ell(w)$.

The (right) weak order on $W$ is the partial order with $u \geq v$ if $\ell(u)=\ell(v)+$ $\ell\left(v^{-1} u\right)$. We write Hasse $(W)$ for the Hasse quiver of the weak order on $W$. The arrows of $\operatorname{Hasse}(W)$ are all arrows $w s_{i} \rightarrow w$ such that $w \in W, s_{i} \in S$, and $\ell\left(w s_{i}\right)>$ $\ell(w)$, or equivalently $\ell\left(w s_{i}\right)=\ell(w)+1$. For $w \in W$, we denote by $w^{+}$(respectively, $\left.w^{-}\right)$the set of arrows in Hasse $(W)$ ending (respectively, starting) at $w$.

The weak order on $W$ is a finite lattice. In particular, it has a maximal element, denoted $w_{0}$ and often called the longest element of $W$. For our purposes, the most important properties of the weak order are the following.

Theorem 2.6. $W$ is congruence uniform [CLM] and polygonal [R3, Theorem 103.7 . 
2.3. Algebraic preliminaries. Fix a base field $k$, and let $A$ be a finite-dimensional $k$-algebra. We write mod $A$ for the finite-dimensional left $A$-modules.

We say that a full subcategory $\mathscr{T}$ of $\bmod A$ is a torsion class if it is closed under factors, isomorphisms and extensions. Torsion-free classes are defined dually. We write tors $A$ for the torsion classes of $A$, and torf $A$ for its torsion-free classes. We view tors $A$ and torf $A$ as posets under the inclusion order. Then we have an antiisomorphism given by $\mathscr{T} \mapsto \mathscr{T}^{\perp}=\left\{X \in \bmod A \mid \operatorname{Hom}_{A}(\mathscr{T}, X)=0\right\}$

$$
\text { tors } A \rightarrow \operatorname{torf} A \text {, }
$$

whose inverse is given by $\mathscr{F} \mapsto{ }^{\perp} \mathscr{F}=\left\{X \in \bmod A \mid \operatorname{Hom}_{A}(X, \mathscr{F})=0\right\}$.

We recall that a torsion class $\mathscr{T}$ of $\bmod A$ is functorially finite if there exists $M \in \bmod A$ such that $\mathscr{T}=$ Fac $M$, where Fac $M$ is the full subcategory of $\bmod A$ consisting of factor modules of finite direct sums of copies of $M$ [AS. We denote by f-tors $A$ (respectively, f-torf $A$ ) the set of all functorially finite torsion (respectively, torsionfree) classes in $\bmod A$. We view $\mathrm{f}$-tors $A$ also as a poset under inclusion. The above anti-isomorphism restricts to an anti-isomorphism f-tors $A \rightarrow \mathrm{f}$-torf $A$.

There is a bijection between f-tors $A$ and a certain class of $A$-modules. Recall that a module $M \in \bmod A$ is called $\tau$-rigid if $\operatorname{Hom}_{A}(M, \tau M)=0$, and $\tau^{-}$-rigid if $\operatorname{Hom}_{A}\left(\tau^{-} M, M\right)=0$. A module $M \in \bmod A$ is called $\tau$-tilting if it is $\tau$-rigid and $|M|=|A|$ holds, where $|M|$ is the number of non-isomorphic indecomposable direct summands of $M$. A module $M \in \bmod A$ is called support $\tau$-tilting if there exists an idempotent $e$ of $A$ such that $M$ is a $\tau$-tilting $(A /\langle e\rangle)$-module. We denote by $\mathrm{s} \tau$-tilt $A$ the set of isomorphism classes of basic support $\tau$-tilting $A$-modules, and by i $\tau$-rigid $A$ (respectively, i $\tau^{-}$-rigid $A$ ) the set of isomorphism classes of indecomposable $\tau$-rigid (respectively, $\tau^{-}$-rigid) $A$-modules. (See $\mathrm{AIR}$ for more background on these notions.) By [AIR, 2.7], we have a surjection $\{\tau$-rigid $A$-modules $\} \rightarrow$ f-tors $A$ given by $M \mapsto \operatorname{Fac} M$, which induces a bijection

$$
\text { s } \tau \text {-tilt } A \stackrel{\sim}{\longrightarrow} \text { f-tors } A .
$$

Recall that $A$ is $\tau$-tilting finite if s $\tau$-tilt $A$ is a finite set, or equivalently, i $\tau$-rigid $A$ is a finite set. It is shown in [DIJ] and [RTT, 0.2] that the following conditions are equivalent.

- $A$ is $\tau$-tilting finite

- $\mathrm{f}$-tors $A$ is a finite set.

- f-tors $A$ (respectively, f-torf $A$ ) forms a complete lattice.

- f-tors $A=\operatorname{tors} A$.

Via the bijection between $\mathrm{f}$-tors $A$ and $\mathrm{s} \tau$-tilt $A$, we obtain a partial order on $\mathrm{s} \tau$-tilt $A$. We refer to the poset on s $\tau$-tilt $A$ as generation order. The arrows of the Hasse diagram of this poset are mutations (see [AIR, Theorem 0.6]).

Using $\tau$-tilting theory, we have the following description of join-irreducible elements in tors $A$.

Theorem 2.7. Let $A$ be a finite dimensional $k$-algebra which is $\tau$-tilting finite. Then we have a bijection given by $M \mapsto \operatorname{Fac} M$

$$
\text { i } \tau \text {-rigid } A \rightarrow \mathrm{j} \text {-Irr(tors } A) \text {. }
$$

Proof. Since $A$ is $\tau$-tilting finite, we have s $\tau$-tilt $A \simeq \operatorname{tors} A$. For $\mathscr{T} \in \operatorname{tors} A$, we take $M=M_{1} \oplus \cdots \oplus M_{n} \in \mathrm{s} \tau$-tilt $A$ such that $\mathscr{T}=$ Fac $M$. It is shown in AIR] that adjacent vertices to $\mathscr{T}$ in the Hasse quiver of tors $A$ are given by Fac $\mu_{k}(M)$ for $1 \leq k \leq n$, where $\mu_{k}(M)$ is the mutation of $M$ at $M_{k}$. Clearly Fac $\mu_{k}(M) \subset \mathscr{T}$ holds if and only if $M_{k} \in \operatorname{Fac}\left(M / M_{k}\right)$. Therefore $\mathscr{T}$ is join-irreducible if and only if there exists a unique $k$ satisfying Fac $\mu_{k}(M) \subset \mathscr{T}$. This is equivalent to $\mathscr{T}=$ Fac $M_{k}$. Since $M_{k}$ is an indecomposable $\tau$-rigid $A$-module, we have the assertion. 
For a complete lattice $L$, we denote by $c j-\operatorname{Irr} L$ the set of completely join-irreducible elements, that is, elements $a \in L$ such that $a=\bigvee S$ for a subset $S$ of $L$ implies $a \in S$. If we drop the $\tau$-tilting finiteness assumption on $A$, then we still have a bijection i $\tau$-rigid $A \rightarrow$ f-tors $A \cap \mathrm{cj}$-Irr(tors $A$ ) given by $M \mapsto$ Fac $M$. The proof is the same, we only need to use [DIJ, Theorem 3.1]. Note that completely joinirreducible elements in tors $A$ are not necessarily functorially finite. For example, consider $\bmod k Q$ for a Kronecker quiver $Q$. Then all preinjective modules together with one tube form such a torsion class.

We denote by brick $A$ the set of isomorphism classes of bricks of $A$. A full subcategory $\mathscr{W}$ of $\bmod A$ is called wide if it is closed under kernels, cokernels and extensions. In this case, $\mathscr{W}$ forms an abelian category and the inclusion functor $\mathscr{W} \rightarrow \bmod A$ is exact. A wide subcategory $\mathscr{W}$ is called local if it contains a unique simple object up to isomorphism. We denote by I-wide $A$ the set of local wide subcategories of $\bmod A$. We have the following easy observation.

Proposition 2.8. Let $A$ be a finite dimensional $k$-algebra. Then we have a bijection brick $A \rightarrow$ I-wide $A$ given by $S \mapsto$ Filt $S$,

where Filt $S$ consists of $A$-modules $X$ which have a filtration $X=X_{0} \supset X_{1} \supset \cdots \supset$ $X_{\ell-1} \supset X_{\ell}=0$ with $\ell \geq 0$ such that $X_{i} / X_{i+1} \simeq S$ for any $0 \leq i<\ell$.

Proof. Let $S$ be a brick of $A$. Then Filt $S$ is a wide subcategory by [Ri, 1.2]. Clearly Filt $S$ has a unique simple object in $S$. Conversely, let $\mathscr{W}$ be a local wide subcategory of $\bmod A$ with a simple object $S$. Clearly $S$ is a brick and we have $\mathscr{W}=$ Filt $S$.

2.4. Preliminaries on preprojective algebras. Let $\Pi=\Pi(W)$ be a preprojective algebra of the same Dynkin type as $W$. To construct $\Pi$, take the Dynkin diagram and replace each edge by a pair of opposite arrows $a, a^{*}$ to obtain the quiver $\bar{Q}$. Then $\Pi(W)$ is the path algebra of $\bar{Q}$ modulo the ideal generated by $\sum_{a}\left(a a^{*}-a^{*} a\right)$. It is a finite-dimensional self-injective algebra. We write $S_{i}$ for the simple module corresponding to vertex $i$.

We let $e_{i}$ be the idempotent corresponding to the vertex $i$. Let $I_{i}$ be the twosided ideal $\Pi\left(1-e_{i}\right) \Pi$. It is maximal as a left ideal and as a right ideal. For each $w \in W$, we take a reduced word $w=s_{i_{1}} \ldots s_{i_{k}}$, and we define

$$
I(w)=I_{i_{1}} \ldots I_{i_{k}}, \mathrm{~T}(w):=\operatorname{Fac} I(w) \text { and } \mathrm{F}(w):=\operatorname{Sub}(\Pi / I(w)) .
$$

Here Sub $X$ refers to the subcategory consisting of subobjects of direct sums of copies of $X$.

The following result due to Mizuno is fundamental.

Theorem 2.9. Mi, 2.14, 2.21]

(a) $I(w)$ does not depend on the choice of the reduced word for $w$.

(b) We have bijections given by $w \mapsto I(w) \mapsto \mathrm{T}(w)$ :

$$
W \stackrel{\sim}{\longrightarrow} \mathrm{s} \tau \text {-tilt } \Pi \stackrel{\sim}{\longrightarrow} \text { tors } \Pi \text {. }
$$

(c) The bijection above from $W$ to tors $\Pi$ is an anti-isomorphism from weak order on $W$ to inclusion order on torsion classes.

We remark that Mizuno uses right modules, while we use left modules, and therefore his results need to be suitably translated to account for this difference. (In particular, this has the effect that he uses left weak order while we use right weak order.) We also remark here that AIR] uses right modules, but writes $I_{w}$ for the the ideal which we would refer to as $I_{w^{-1}}$.

Thus $I(e)=\Pi$ gives the maximum torsion class $\mathrm{T}(e)=\bmod \Pi$ and $I\left(w_{0}\right)=0$ gives the minimum torsion class $\mathrm{T}\left(w_{0}\right)=\{0\}$, where $e \in W$ is the identity and $w_{0} \in W$ is the longest element. 

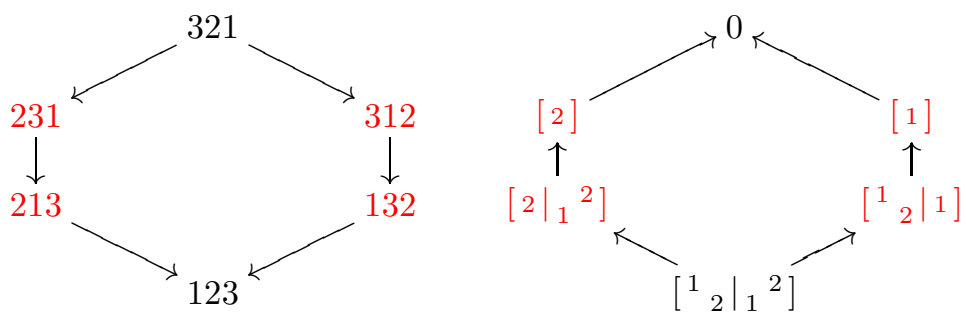

Figure 5. The weak order and $\mathrm{s} \tau$-tilt $\Pi$ in type $A_{2}$

The following is an easy consequence of (2.1).

Lemma 2.10. Let $w, v \in W$. Then $\ell(w v)=\ell(w)+\ell(v)$ holds if and only if $I(w v)=$ $I(w) I(v)$ holds. If this holds, then we have $I(w v)=I(w) I(v)=I(w) \otimes_{\Pi} I(v)$.

We show a few examples. Here, ${ }^{1} 2_{3}$, for example, refers to the indecomposable $\bar{\Pi}$-module with composition factors $S_{1}, S_{2}, S_{3}$ from top to bottom.

Example 2.11. The left picture in Figure 5 shows the weak order on permutations in $S_{3}$ (the Weyl group of type $A_{2}$ ). The right picture in Figure 5 shows the set s $\tau$-tilt $\Pi$ for $\Pi$ of type $A_{2}$, arranged in generation order. We show in red the meetirreducible elements of $W$ and the join-irreducible elements of $\mathbf{s} \tau$-tilt $\Pi$. The two pictures are arranged so that the map $w \rightarrow I(w)$ is accomplished by a translation of the page.

Example 2.12. Figure 6 shows the weak order on permutations in $S_{4}$ (the Weyl group of type $A_{3}$ ) and $\mathrm{s} \tau$-tilt $\Pi$ for $\Pi$ of type $A_{3}$, with the same conventions as described in Example 2.11.

\section{HomologicAl CHARACTERIZATIONS OF LAYERS}

In this section we prove Theorem 1.2, the homological characterization of layer modules of the preprojective algebra $\Pi$. The assertion that every layer module is a stone was shown in AIRT, 2.3]. It is also easy to show that the stones of $\Pi$ are exactly the $\Pi$-modules which are 2 -spherical as $\widehat{\Pi}$-modules. Indeed, for any $\Pi$-module $L$, we have $\operatorname{Ext}_{\widehat{\Pi}}^{1}(L, L)=\operatorname{Ext}_{\Pi}^{1}(L, L)$ and $\operatorname{Ext}_{\widehat{\Pi}}^{2}(L, L)=D \operatorname{End}_{\Pi}(L)$, and the assertion follows.

We show that any brick $L$ of $\Pi$ is a stone. Let $\langle-,-\rangle$ be the Euler form on $K_{0}(\mathrm{fd} \widehat{\Pi})$. For $x=\left(x_{i}\right)_{i \in Q_{0}} \in \mathbb{Z}^{Q_{0}}$, we have

$$
\langle x, x\rangle=2\left(\sum_{i \in Q_{0}} x_{i}^{2}-\sum_{(i \rightarrow j) \in Q_{1}} x_{i} x_{j}\right),
$$

which is a non-negative even integer. Since the restriction of $\langle-,-\rangle$ to $K_{0}(\bmod \Pi)$ is positive definite, we have

$$
2-\operatorname{dim}_{k} \operatorname{Ext}_{\Pi}^{1}(L, L)=\sum_{i=0}^{2}(-1)^{i} \operatorname{dim}_{k} \operatorname{Ext}_{\Pi}^{i}(L, L)=\langle\underline{\operatorname{dim}} L, \underline{\operatorname{dim}} L\rangle \geq 2,
$$

which implies $\operatorname{Ext}_{\Pi}^{1}(L, L)=0$. Thus $L$ is a stone.

To complete the proof, we will show that every stone is a layer. As before, $\mathrm{D}^{\mathrm{b}}(\mathrm{fd} \widehat{\Pi})$ is the bounded derived category of finite dimensional $\widehat{\Pi}$-modules. Let $\widehat{I_{i}}:=\widehat{\Pi}\left(1-e_{i}\right) \widehat{\Pi}$ be an ideal of $\widehat{\Pi}$. Then we have an autoequivalence

$$
F_{i}:=\widehat{I}_{i} \stackrel{\mathrm{L}}{\otimes_{\widehat{\Pi}}}-: \mathrm{D}^{\mathrm{b}}(\mathrm{fd} \widehat{\Pi}) \rightarrow \mathrm{D}^{\mathrm{b}}(\mathrm{fd} \widehat{\Pi})
$$



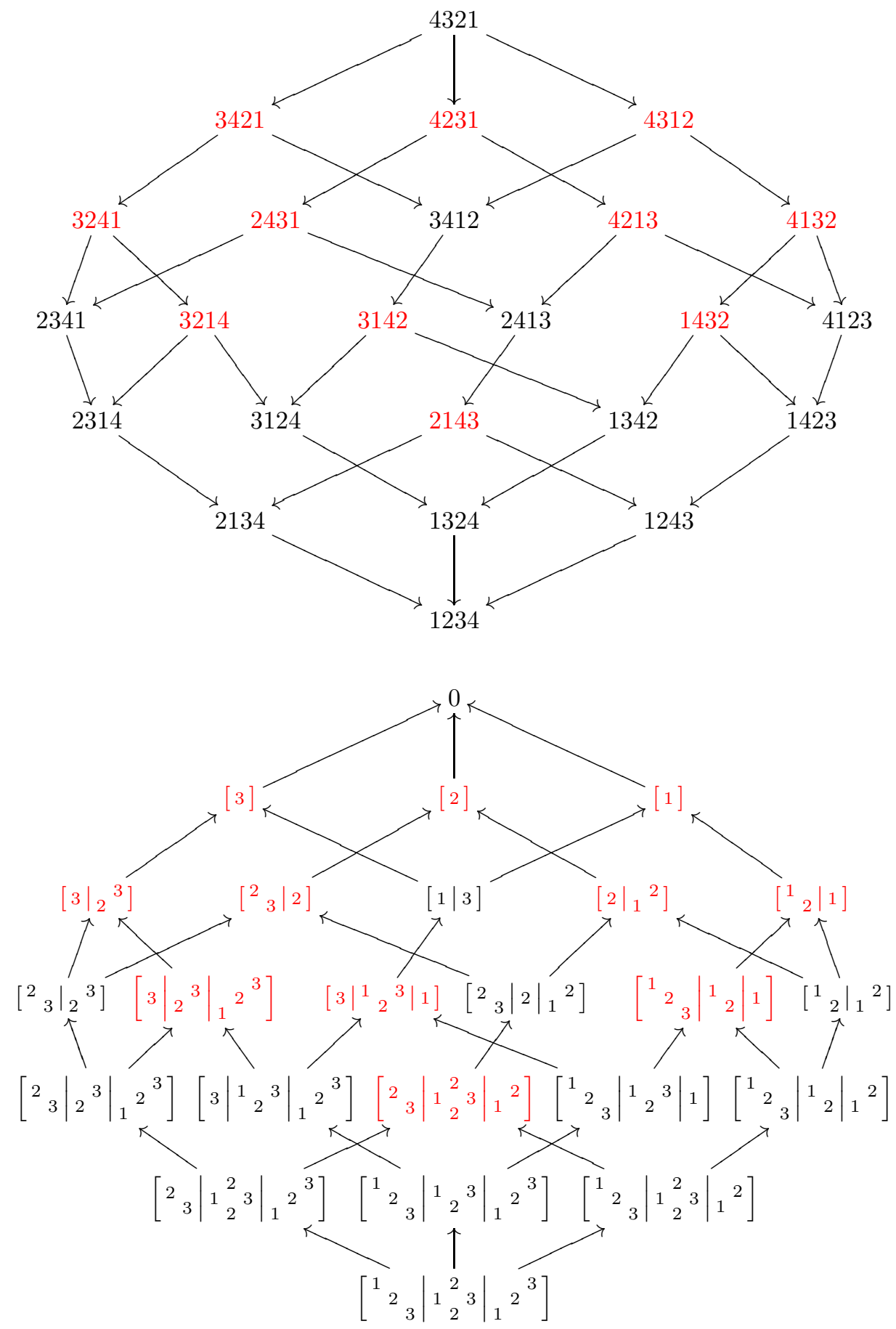

FiguRE 6 . The weak order and $\mathrm{s} \tau$-tilt $\Pi$ in type $A_{3}$

with quasi-inverse

$$
F_{i}^{-1}:=\operatorname{RHom}_{\widehat{\Pi}}\left(\widehat{I}_{i},-\right): \mathrm{D}^{\mathrm{b}}(\mathrm{fd} \widehat{\Pi}) \rightarrow \mathrm{D}^{\mathrm{b}}(\mathrm{fd} \widehat{\Pi}) .
$$

We identify $\Pi$-modules with $\widehat{\Pi}$-modules annihilated by $e_{0}$, where $e_{0}$ is an idempotent of $\widehat{\Pi}$ satisfying $\Pi=\widehat{\Pi} /\left\langle e_{0}\right\rangle$. 
The following assertions are easy to check.

Lemma 3.1. Let $L$ be a finite-dimensional П-module.

(a) We have $I_{i}=\widehat{I}_{i} \otimes_{\widehat{\Pi}} \Pi=\Pi \otimes_{\widehat{\Pi}} \widehat{I}_{i}$.

(b) We have $I_{i} \otimes_{\Pi} L=\widehat{I}_{i} \otimes_{\widehat{\Pi}} L$ and $\operatorname{Hom}_{\Pi}\left(I_{i}, L\right)=\operatorname{Hom}_{\widehat{\Pi}}\left(\widehat{I}_{i}, L\right)$.

The following observation plays a key role.

Proposition 3.2. Let $L$ be a stone of $\Pi$ and $i$ a vertex of $\bar{Q}$.

(a) If $L \neq S_{i}$, then at least one of $\operatorname{Hom}_{\Pi}\left(S_{i}, L\right)=0$ or $\operatorname{Hom}_{\Pi}\left(L, S_{i}\right)=0$ holds.

(b) If $\operatorname{Hom}_{\Pi}\left(S_{i}, L\right)=0$, then $F_{i}(L) \simeq I_{i} \otimes_{\Pi} L$ is a stone of $\Pi$.

(c) If $\operatorname{Hom}_{\Pi}\left(L, S_{i}\right)=0$, then $F_{i}^{-1}(L) \simeq \operatorname{Hom}_{\Pi}\left(I_{i}, L\right)$ is a stone of $\Pi$.

Proof. We already observed that stones of $\Pi$ are precisely $\Pi$-modules which are 2-spherical as $\widehat{\Pi}$-modules.

(a) If both $\operatorname{Hom}_{\Pi}\left(S_{i}, L\right)$ and $\operatorname{Hom}_{\Pi}\left(L, S_{i}\right)$ are non-zero, the composition $L \rightarrow$ $S_{i} \rightarrow L$ is a non-zero non-isomorphic endomorphism of $L$. This is a contradiction since $\operatorname{End}_{\Pi}(L)=k$.

(b) Since $F_{i}$ is an autoequivalence of $\mathrm{D}^{\mathrm{b}}(\mathrm{fd} \widehat{\Pi})$, we have that $F_{i}(L)$ is a 2 -spherical object. We show that it is a $\widehat{\Pi}$-module, that is, $\operatorname{Tor}_{j}^{\widehat{\Pi}}\left(\widehat{I}_{i}, L\right)=0$ holds for any $j \neq 0$. Since the $\widehat{\Pi}$-module $\widehat{I}_{i}$ has projective dimension at most one BIRS, we only have to check the case $j=1$. Since

$$
\operatorname{Tor}_{1}^{\widehat{\Pi}}\left(\widehat{I}_{i}, L\right) \simeq \operatorname{Tor}_{2}^{\widehat{\Pi}}\left(S_{i}, L\right) \simeq D \operatorname{Ext}_{\widehat{\Pi}}^{2}\left(L, S_{i}\right) \simeq \operatorname{Hom}_{\widehat{\Pi}}\left(S_{i}, L\right)=0
$$

holds by our assumption, we have the assertion. Thus we have $F_{i}(L) \simeq \widehat{I}_{i} \otimes_{\widehat{\Pi}} L$, which is isomorphic to $I_{i} \otimes_{\Pi} L$ by Lemma 3.1 .

(c) Similar to (b).

We need the following observation.

Lemma 3.3. Let $w \in W$ and let $L$ be a stone of $\Pi$ which belongs to $\mathrm{T}(w)$. For any $i$ satisfying $\operatorname{Hom}_{\Pi}\left(L, S_{i}\right) \neq 0$, the following assertions hold.

(a) $\ell\left(s_{i} w\right)>\ell(w)$ and $I\left(s_{i} w\right)=I_{i} I(w)=I_{i} \otimes_{\Pi} I(w)$.

(b) If $L \not S_{i}$, then $\operatorname{Hom}_{\Pi}\left(S_{i}, L\right)=0$ and $F_{i}(L)$ is a stone of $\Pi$ which belongs to $\mathrm{T}\left(s_{i} w\right)$.

Proof. (a) Since $L \in \mathrm{T}(w)$ and $\operatorname{Hom}_{\Pi}\left(L, S_{i}\right) \neq 0$, we have $\operatorname{Hom}_{\Pi}\left(I(w), S_{i}\right) \neq 0$. Thus $I_{i} I(w) \neq I(w)$ holds. Thus we have the assertions by Lemma 2.10.

(b) By Proposition 3.2 , $\operatorname{Hom}_{\Pi}\left(S_{i}, L\right)=0$ holds, and $F_{i}(L)$ is a stone of $\Pi$. Since we have a surjection $I(w)^{\oplus m} \rightarrow L$, we have a surjection $I\left(s_{i} w\right)^{\oplus m}=I_{i} \otimes_{\Pi}$ $I(w)^{\oplus m} \rightarrow I_{i} \otimes_{\Pi} L=F_{i}(L)$. Thus $F_{i}(L)$ belongs to $\mathrm{T}\left(s_{i} w\right)$.

For $w \in W$, we have an ideal $\widehat{I}(w)$ of $\widehat{\Pi}$ satisfying $\widehat{I}(w) \supseteq\left\langle e_{0}\right\rangle$ and $\widehat{I}(w) /\left\langle e_{0}\right\rangle=$ $I(w)$. Let

$$
F(w):=\widehat{I}(w) \stackrel{\mathrm{L}}{\otimes_{\widehat{\Pi}}}-: \mathrm{D}^{\mathrm{b}}(\mathrm{fd} \widehat{\Pi}) \rightarrow \mathrm{D}^{\mathrm{b}}(\mathrm{fd} \widehat{\Pi}) .
$$

For any reduced expression $w=s_{i_{1}} \cdots s_{i_{\ell}}$, we have

$$
\widehat{I}(w)=\widehat{I}_{i_{1}} \cdots \widehat{I}_{i_{\ell}} \text { and } F(w)=F_{i_{1}} \circ \cdots \circ F_{i_{\ell}} .
$$

Lemma 3.4. Let $L$ be a stone of $\Pi$. Then there exists $v \in W$ such that $F(v)(L)$ is a simple $\Pi$-module $S_{i}$.

Proof. Assume that $L$ is not simple. Taking $i_{1}$ such that $\operatorname{Hom}_{\Pi}\left(L, S_{i_{1}}\right) \neq 0$ and applying Lemma 3.3 to the stone $L \in \bmod \Pi=\mathrm{T}(e)$, we have that $F_{i_{1}}(L)$ is a stone in $\operatorname{Fac} I_{i_{1}}$. 
Assume that $F_{i_{1}}(L)$ is not simple. Taking $i_{2}$ such that $\operatorname{Hom}_{\Pi}\left(F_{i_{1}}(L), S_{i_{2}}\right) \neq 0$ and applying Lemma 3.3 to the stone $F_{i_{1}}(L) \in \mathrm{Fac} I_{i_{1}}$, we have that $F_{i_{2}} F_{i_{1}}(L)$ is a stone in $\operatorname{Fac}\left(I_{i_{2}} I_{i_{1}}\right)$ and $\ell\left(s_{i_{2}} s_{i_{1}}\right)=2$ holds.

We repeat this process. Since the lengths of elements in $W$ are bounded by $\ell\left(w_{0}\right)$, the process must stop, that is, there exists $v \in W$ such that $F(v)(L)$ is a simple П-module $S_{i}$.

We need the following general observation.

Lemma 3.5. For any $v, w \in W$ satisfying $I(v) \supset I(w)$, we have an isomorphism $D(I(v) / I(w)) \simeq I\left(w_{0} w^{-1}\right) / I\left(w_{0} v^{-1}\right)$ of $\Pi$-modules.

Proof. Applying $D$ to the exact sequence $0 \rightarrow I(w) \rightarrow I(v) \rightarrow I(v) / I(w) \rightarrow 0$ of $\Pi^{\mathrm{op}}$-modules, we have an exact sequence

$$
0 \rightarrow D(I(v) / I(w)) \rightarrow D(I(v)) \rightarrow D(I(w)) \rightarrow 0
$$

of $\Pi$-modules. On the other hand, we have an exact sequence

$$
0 \rightarrow I\left(w_{0} w^{-1}\right) / I\left(w_{0} v^{-1}\right) \rightarrow \Pi / I\left(w_{0} v^{-1}\right) \rightarrow \Pi / I\left(w_{0} w^{-1}\right) \rightarrow 0
$$

of $\Pi$-modules. Using an isomorphism $D(I(w)) \simeq \Pi / I\left(w_{0} w^{-1}\right)$ of $\Pi$-modules for any $w \in W$ ORT and comparing (3.1) and (3.2), we have the assertion.

Now we are ready to finish the proof of Theorem 1.2

Proof of Theorem 1.2. As shown in the first paragraph of this section, we can complete the proof by showing that every stone is a layer. Let $L$ be a stone of $\Pi$. By Lemma 3.4 there exists $v \in W$ such that $F(v)(L)=\widehat{I}(v) \otimes_{\widehat{\Pi}}^{\mathbf{L}} L$ is a simple $\Pi$-module $S_{i}$. Thus $L$ equals

$$
\begin{aligned}
& \operatorname{RHom}_{\widehat{\Pi}}\left(\widehat{I}(v), S_{i}\right)=\operatorname{Hom}_{\widehat{\Pi}}\left(\widehat{I}(v), S_{i}\right) \stackrel{\operatorname{Lem} 3.1}{=} \operatorname{Hom}_{\Pi}\left(I(v), S_{i}\right)=D\left(D S_{i} \otimes_{\Pi} I(v)\right) \\
& =D\left(\left(\Pi / I_{i}\right) \otimes_{\Pi} I(v)\right)=D\left(I(v) / I_{i} I(v)\right) \stackrel{\operatorname{Lem} \underline{\underline{2.10}}}{=} D\left(I(v) / I\left(s_{i} v\right)\right) .
\end{aligned}
$$

This is a layer of $\Pi$ by Lemma 3.5

\section{Bijections: Theorems 1.1 And 1.3}

Throughout this section, let $\Pi$ be a preprojective algebra of Dynkin type and $W$ the corresponding Weyl group. The main result of this section is that a certain diagram is commutative. Commutativity of that diagram includes Theorems 1.1 and 1.3 .

For an arrow $a: w s_{i} \rightarrow w$ in $H$, we have a natural inclusion $I(w) \supset I\left(w s_{i}\right)$ of ideals of $\Pi$, and we associate to $a$ the $\Pi$-module

$$
L(a):=I(w) / I\left(w s_{i}\right)=\left(I(w) e_{i}\right) /\left(I(w) I_{i} e_{i}\right),
$$

where the right equality follows from $I(w)=I(w) e_{i} \oplus I(w)\left(1-e_{i}\right), I\left(w s_{i}\right)=$ $I(w) I_{i} e_{i} \oplus I(w) I_{i}\left(1-e_{i}\right)$ and $I_{i}\left(1-e_{i}\right)=\Pi\left(1-e_{i}\right)$. We also define maps $L$ : $\mathrm{m}$-Irr $W \rightarrow$ layer $\Pi$ and $M: \mathrm{m}$-Irr $W \rightarrow \mathrm{i} \tau$-rigid $\Pi$ as

$$
L(m):=L(a) \text { and } M(m):=I(m) e_{i}
$$

for $m \in \mathrm{m}-\operatorname{Irr} W$ and $a: m^{*}=m s_{i} \rightarrow m$ in $H$.

Theorem 4.1. Let $\Pi$ be a preprojective algebra of Dynkin type and $W$ the corresponding Weyl group. Then we have the commutative diagram shown in Figure 3 . The maps are bijections or surjections as marked with tildes " " or double-headed arrows.

We start with the following simple observation. 
Lemma 4.2. $L(m)=M(m) / \operatorname{rad} M(m)_{\operatorname{End}_{\Pi}(M(m))}$ holds for any $m \in \mathrm{m}$-Irr $W$.

Proof. We have $M(m)=I(m) e_{i}$ and $L(m)=I(m) e_{i} / I(m) I_{i} e_{i}$. Thus we only have to show $I(m) I_{i} e_{i}=\left(I(m) e_{i}\right) \operatorname{rad}_{\operatorname{End}}\left(I(m) e_{i}\right)$.

Since $\mathrm{T}(m)=\operatorname{Fac} I(m)$ is join-irreducible, we have $I(m) \in \operatorname{Fac}\left(I(m) e_{i}\right)$. Thus

$$
\begin{aligned}
\left(I(m) e_{i}\right) \operatorname{rad}_{\operatorname{End}_{\Pi}\left(I(m) e_{i}\right)} & =\sum_{f \in \operatorname{rad}_{\operatorname{End}_{\Pi}\left(I(m) e_{i}\right)} \operatorname{Im}\left(f: I(m) e_{i} \rightarrow I(m) e_{i}\right)} \operatorname{Im}\left(g: I(m) \rightarrow I(m) e_{i}\right),
\end{aligned}
$$

where $\operatorname{rad}_{\Pi}$ is the radical of the category $\bmod \Pi$ and hence $\operatorname{rad}_{\Pi}\left(I(m), I(m) e_{i}\right)$ consists of morphisms which are not split epimorphisms.

By [Mi, Lemma 2.7], we have a surjection $\Pi \rightarrow \operatorname{End}_{\Pi}(I(m))$ given by $x \mapsto$ $(y \mapsto y x)$. This induces surjections $\Pi e_{i} \rightarrow \operatorname{Hom}_{\Pi}\left(I(m), I(m) e_{i}\right)$ and $I_{i} e_{i} \rightarrow$ $\operatorname{rad}_{\Pi}\left(I(m), I(m) e_{i}\right)$. Therefore we have

$$
\sum_{g \in \operatorname{rad}_{\Pi}\left(I(m), I(m) e_{i}\right)} \operatorname{Im}\left(g: I(m) \rightarrow I(m) e_{i}\right)=I(m) I_{i} e_{i},
$$

which completes the proof.

In Section 2.1 (particularly Theorem 2.3 and Corollary 2.4), we described how the forcing order on join-irreducible elements interacts with the polygons (squares and rectangles) in $W$. One ingredient in the proof of Theorem 4.1 is a similar description of how the layer labelling interacts with polygons.

Recall from the introduction that a pair of layer modules $X, Y$ form a doubleton if $\operatorname{Ext}_{\Pi}^{1}(Y, X)$ and $\operatorname{Ext}_{\Pi}^{1}(X, Y)$ are one-dimensional, and the corresponding extensions are again layer modules. Recall also that the doubleton extension order on layer modules is the transitive closure of the relation with $A>B$ if there exists a doubleton $A, C$ such that $B$ is the extension of $A$ by $C$ or of $C$ by $A$.

Proposition 4.3. For a polygon in $W$ (necessarily a square or hexagon), the layer labelling has the following configuration:
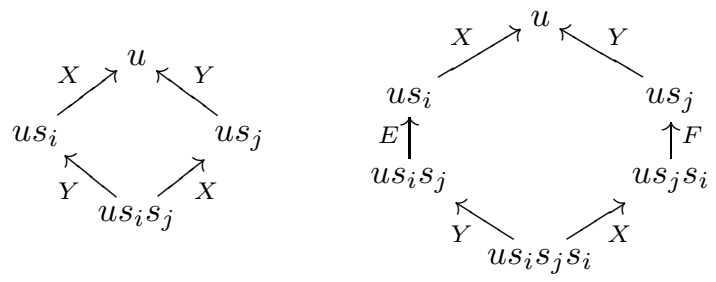

Moreover, in the hexagon case the layers $X$ and $Y$ form a doubleton and there exist short exact sequences of П-modules:

$$
0 \rightarrow X \rightarrow E \rightarrow Y \rightarrow 0 \text { and } 0 \rightarrow Y \rightarrow F \rightarrow X \rightarrow 0
$$

Thus $X \geq E, X \geq F, Y \geq E$, and $Y \geq F$ in the doubleton extension order.

Proof. Of the two diagrams in the statement of the theorem, the hexagon occurs if and only if $i$ and $j$ are neighbouring in $\bar{Q}$. We argue the hexagon case. The square case is similar but simpler. What we need to show is the following:

(i) There are isomorphisms of П-modules:

$$
I(u) / I(u) I_{i} \simeq I(u) I_{j} I_{i} / I(u) I_{i} I_{j} I_{i} \text { and } I(u) / I(u) I_{j} \simeq I(u) I_{i} I_{j} / I(u) I_{i} I_{j} I_{i} \text {. }
$$

(ii) There are short exact sequences of $\Pi$-modules:

$$
\begin{gathered}
0 \rightarrow I(u) / I(u) I_{i} \rightarrow I(u) I_{i} / I(u) I_{i} I_{j} \rightarrow I(u) I_{i} I_{j} / I(u) I_{i} I_{j} I_{i} \rightarrow 0, \\
0 \rightarrow I(u) / I(u) I_{j} \rightarrow I(u) I_{j} / I(u) I_{j} I_{i} \rightarrow I(u) I_{j} I_{i} / I(u) I_{i} I_{j} I_{i} \rightarrow 0 .
\end{gathered}
$$


We first show (i). The partial order tells us, by Lemma 2.10 that we have

$$
I(u) / I(u) I_{i}=I(u) \otimes_{\Pi}\left(\Pi / I_{i}\right) \text { and } I(u) I_{j} I_{i} / I(u) I_{i} I_{j} I_{i}=I(u) \otimes_{\Pi}\left(I_{j} I_{i} / I_{i} I_{j} I_{i}\right) .
$$

Since $\Pi / I_{i} \simeq S_{i} \simeq I_{j} I_{i} / I_{i} I_{j} I_{i}$ holds by an easy calculation for the preprojective algebra $\Pi / I_{i} I_{j} I_{i}$ of type $A_{2}$ (see Figure 5 ), we have

$$
I(u) / I(u) I_{i}=I(u) \otimes_{\Pi}\left(\Pi / I_{i}\right) \simeq I(u) \otimes_{\Pi}\left(I_{j} I_{i} / I_{i} I_{j} I_{i}\right)=I(u) I_{j} I_{i} / I(u) I_{i} I_{j} I_{i} .
$$

The other isomorphism follows by interchanging $i$ and $j$.

Now we show (ii). Again by an easy calculation for the preprojective algebra $\Pi / I_{i} I_{j} I_{i}$ of type $A_{2}$ (see Figure [5), we have $\Pi / I_{i} \simeq S_{i}, I_{i} / I_{i} I_{j} \simeq\left(\begin{array}{c}S_{j} \\ S_{i}\end{array}\right)$ and $I_{i} I_{j} / I_{i} I_{j} I_{i} \simeq S_{j}$. Thus there exists an exact sequence

$$
0 \rightarrow \Pi / I_{i} \rightarrow I_{i} / I_{i} I_{j} \rightarrow I_{i} I_{j} / I_{i} I_{j} I_{i} \rightarrow 0
$$

of $\Pi$-modules. Applying $I(u) \otimes_{\Pi}-$, we obtain the first sequence. The second one follows by interchanging $i$ and $j$.

We verify that $X$ and $Y$ form a doubleton in the hexagon case. If the labels are simple, then the extension groups are certainly one-dimensional, and the extensions are layers. Any hexagon is obtained by applying $I(u) \otimes_{\Pi}-$ to such a hexagon, as above. This implies the desired result once we note that, by Lemma 3.1, we can instead consider applying $\widehat{I}(u) \stackrel{\mathrm{L}}{\otimes_{\widehat{\Pi}}}-$, which is an auto-equivalence of $\mathrm{D}^{\mathrm{b}}(\mathrm{fd} \widehat{\Pi})$ by BIRS.

Now $X \geq E, X \geq F, Y \geq E$, and $Y \geq F$ because there exist short exact sequences $0 \rightarrow X \rightarrow E \rightarrow Y \rightarrow 0$ and $0 \rightarrow Y \rightarrow F \rightarrow X \rightarrow 0$.

The ideas in the proof above also lead to the following lemma. For vertices $i \neq j$ in $\bar{Q}$, let $W_{i, j}:=\left\langle s_{i}, s_{j}\right\rangle \subset W$ be a parabolic subgroup of $W$. For $w \in W$, the coset $w W_{i, j}$ is an interval in the weak order on $W$. We write $\left.H\right|_{w W_{i, j}}$ for the restriction of $\operatorname{Hasse}(W)$ to $w W_{i, j}$. Define $w^{+}$(respectively, $w^{-}$) to be the set of arrows in Hasse $(W)$ starting (respectively, ending) at $w$. For a set $S$ of $\Pi$ modules, we denote by $\mathrm{T}(S)$ (respectively, $\mathrm{F}(S)$ ) the smallest torsion (respectively, torsionfree) class in mod $\Pi$ containing $S$. For convenience and brevity, we will omit set braces inside the operator $\mathrm{T}(\bullet)$, so that, for example $\mathrm{T}\left(L_{0}, M_{i} \mid i \in I\right)$ would mean $\mathrm{T}\left(\left\{L_{0}\right\} \cup\left\{M_{i} \mid i \in I\right\}\right)$. Recall that the layer labelling of Hasse $(W)$ maps each Hasse arrow $x \rightarrow y$ to the isomorphism class of the corresponding concrete layer $L(x \rightarrow y):=I(y) / I(x)$.

Lemma 4.4. If $a: w s_{i} \rightarrow w$ is an arrow in $\operatorname{Hasse}(W)$, and $j \neq i$ is a vertex in $\bar{Q}$, then

$$
\mathrm{T}\left(L(b)\left|b \in w^{-} \cap H\right|_{w W_{i, j}}\right)=\mathrm{T}\left(L(a), L(b)\left|b \in\left(w s_{i}\right)^{-} \cap H\right|_{w W_{i, j}}\right) .
$$

Proof. We have either the square or the hexagon in Proposition 4.3. We argue the hexagon case. The square case is similar but simpler. If $w$ coincides with $u$ in Proposition 4.3, the desired equality reduces to an identity $\mathrm{T}(X, Y)=\mathrm{T}(X, E)$, which follows from the exact sequence $0 \rightarrow X \rightarrow E \rightarrow Y \rightarrow 0$. If $w$ coincides with $u s_{j}$ in Proposition 4.3 the desired equality reduces to an identity $\mathrm{T}(F)=\mathrm{T}(F, X)$, which follows from the exact sequence $0 \rightarrow Y \rightarrow F \rightarrow X \rightarrow 0$. If $w$ coincides with $u s_{i} s_{j}$ in Proposition 4.3, the desired equality reduces to an identity $\mathrm{T}(Y)=\mathrm{T}(Y)$, which clearly holds.

Another ingredient in the proof of Theorem 4.1 is a precise connection between torsion classes and layer modules.

Theorem 4.5. For any $w \in W$, we have

$$
\mathrm{T}(w)=\mathrm{T}\left(L(a) \mid a \in w^{-}\right) \text {and } \mathrm{F}(w)=\mathrm{F}\left(L(a) \mid a \in w^{+}\right) .
$$


Proof. We only prove the first equality since the second one is proved similarly. We use decreasing induction on $W$. The statement is clear for the longest element $w_{0}$ since both sides are $\{0\}$ in this case.

Let $a: w s_{i} \rightarrow w$ be an arrow in $\operatorname{Hasse}(W)$. Assume that the assertion holds for $w s_{i}$, that is,

$$
\mathrm{T}\left(w s_{i}\right)=\mathrm{T}\left(L(b) \mid b \in\left(w s_{i}\right)^{-}\right)
$$

Using obvious decompositions

$$
w^{-}=\bigcup_{j \neq i}\left(\left.w^{-} \cap H\right|_{w W_{i, j}}\right) \text { and }\left(w s_{i}\right)^{-}=\bigcup_{j \neq i}\left(\left.\left(w s_{i}\right)^{-} \cap H\right|_{w W_{i, j}}\right),
$$

we have

$$
\begin{array}{lll}
\mathrm{T}\left(L(b) \mid b \in w^{-}\right) & \stackrel{44.2}{=} & \left.\mathrm{T}\left(\mathrm{T}\left(L(b)\left|b \in w^{-} \cap H\right|_{w W_{i, j}}\right)\right) \mid j \neq i\right) \\
& \stackrel{\text { Lem }}{=} & \left.\mathrm{T}\left(\mathrm{T}\left(L(a), L(b)\left|b \in\left(w s_{i}\right)^{-} \cap H\right|_{w W_{i, j}}\right)\right) \mid j \neq i\right) \\
& \stackrel{44.2}{=} & \mathrm{T}\left(L(a), \mathrm{T}\left(L(b) \mid b \in\left(w s_{i}\right)^{-}\right)\right) \\
& \stackrel{4.17}{=} & \mathrm{T}\left(L(a), \mathrm{T}\left(w s_{i}\right)\right) \\
= & \mathrm{T}(w),
\end{array}
$$

where the last equality follows from having an exact sequence $0 \rightarrow I\left(w s_{i}\right) \rightarrow$ $I(w) \rightarrow L(a) \rightarrow 0$ and $I\left(w s_{i}\right) \in \mathrm{T}(w)$.

Proposition 4.6. Two arrows $x \rightarrow y$ and $x^{\prime} \rightarrow y^{\prime}$ in Hasse $(W)$ have con $(x, y)=$ $\operatorname{con}\left(x^{\prime}, y^{\prime}\right)$ if and only if they have the same layer label.

Proof. Proposition 4.3 implies in particular that the map $(x \rightarrow y) \mapsto I(y) / I(x)$ is constant on components of SFPoly $(W)$. Thus by Corollary 2.5. if $x \rightarrow y$ and $x^{\prime} \rightarrow y^{\prime}$ have $\operatorname{con}(x, y)=\operatorname{con}\left(x^{\prime}, y^{\prime}\right)$, then they have the same layer labelling.

If $x \rightarrow y$ and $x^{\prime} \rightarrow y^{\prime}$ have the same layer labelling, let $m$ be the unique meetirreducible element with $\operatorname{con}\left(m^{*}, m\right)=\operatorname{con}(x, y)$, and similarly let $m^{\prime}$ be the meetirreducible corresponding to $x^{\prime} \rightarrow y^{\prime}$. Then $L\left(m^{*} \rightarrow m\right)=L\left(\left(m^{\prime}\right)^{*} \rightarrow m\right)$, so by Theorem 4.5. $\mathrm{T}(m)=\mathrm{T}\left(L\left(m^{*} \rightarrow m\right)\right)=\mathrm{T}\left(L\left(\left(m^{\prime}\right)^{*} \rightarrow m\right)\right)=\mathrm{T}\left(m^{\prime}\right)$. Since $\mathrm{T}$ : $W \rightarrow$ tors $\Pi$ is a bijection, we see that $m=m^{\prime}$, so that $\operatorname{con}(x, y)=\operatorname{con}\left(x^{\prime}, y^{\prime}\right)$.

Proof of Theorem 4.1. Commutativity of the three triangles involving $\mathrm{m}-\operatorname{Irr}(W)$, $\mathrm{j}-\operatorname{Irr}(W)$, Con $_{\mathrm{Jl}}(W)$, and Hasse $(W)$, and correctness of the markings of the arrows as bijections or surjections was established in Section2.1 in the more general context of congruence uniform lattices. (See especially Figure 4.)

The map from $\mathrm{Hasse}_{1}(W)$ to layer $\Pi$ is surjective by definition. Proposition 4.6. together with the commutativity of the triangles containing Con JI $(W)$, then implies the commutativity of the two triangles containing the map from $\mathrm{Hasse}_{1}(W)$ to layer $\Pi$ and also implies that the map from $\mathrm{m}-\operatorname{Irr}(W)$ to layer $\Pi$ is a bijection. The triangle containing $\mathrm{m}$ - $\operatorname{Irr}(W)$, i $\tau$-rigid $\Pi$ and layer $\Pi$ commutes by Lemma 4.2 , and the left bottom square commutes by Theorem 4.5. The remaining part of the diagram commutes dually. The maps $M: \mathrm{m}$-Irr $W \rightarrow \mathrm{i} \tau$-rigid $\Pi$, Fac : $\mathrm{i} \tau$-rigid $\Pi \rightarrow$ tors $\Pi$ and Filt : brick $\Pi \rightarrow$ I-wide $\Pi$ are bijections by Theorems 2.9, 2.7] and Proposition 2.8, The bijectivity of the other maps follows from commutativity.

The bijections $\mathrm{j}$-Irr $W \simeq$ layer $\Pi \simeq \mathrm{m}$-Irr $W$ given in Theorem 4.1 combined with Proposition 2.2, imply the following corollary:

Corollary 4.7. $\mathrm{T}(w)$ is the smallest subcategory of $\Pi$-mod which is closed under extensions and quotients and contains the layers corresponding to the canonical meet representation of $w . F(w)$ is the smallest subcategory of $\Pi-m o d$ which is closed under 
extensions and subobjects and contains the layers corresponding to the canonical join representation of $w$.

Remark 4.8. Let $a: w s_{i} \rightarrow w$ be an arrow in $H$. One might wonder if $F(w)$ is a unique stone of $\Pi$ which belongs to $\mathrm{T}(w) \backslash \mathrm{T}\left(w s_{i}\right)$.

This has an easy counterexample: let $\Pi$ be of type $A_{2}$, and let $w:=e$ and $s_{i}:=s_{1}$. Then both layer modules $S_{1}$ and $P_{1}$ belong to $(\bmod \Pi) \backslash\left(\operatorname{Fac} I_{1}\right)$.

It therefore seems that $L(a)$ should be characterized by some kind of minimality among the stones of $\mathrm{T}(w) \backslash \mathrm{T}\left(w s_{i}\right)$.

\section{Doubleton EXTENSION ORDER ON LAYER MODUlES}

In this section, we prove Theorem 1.4 and a characterization of the doubleton extension order on layer modules. The last ingredient needed is the following proposition.

Proposition 5.1. Suppose $A, B$, and $C$ are layers of $\Pi$ such that $A, C$ is a doubleton and $B$ is the extension of $C$ by $A$. Then there exists a hexagon in weak order such that the layer ordering of one of its chains is $(A, B, C)$, read either from bottom to top or from top to bottom.

Before proving Proposition 5.1, we show how it completes the proof of Theorem 1.4

Proof of Theorem 1.4. Theorem 4.1 already states that the map $j \mapsto I\left(j_{*}\right) / I(j)$ is a bijection from the set of join-irreducible elements of $W$ to the set of layer modules of $\Pi$. Proposition 4.3 implies that every arrow in the quiver $\operatorname{FPoly}(W)$ (defined in Section 2.1) gives rise to an order relation in the doubleton extension order. Proposition 5.1 shows that each doubleton extension comes from some arrow in FPoly $(W)$. Corollary 2.4 (which applies in light of Theorem 2.6) thus implies that $j \mapsto I\left(j_{*}\right) / I(j)$ is an isomorphism from the forcing order on join-irreducible elements of $W$ to the doubleton extension order on layers.

We now prepare to prove Proposition 5.1

Lemma 5.2. If $X, Y$ is a doubleton, then $\operatorname{Hom}_{\Pi}(X, Y)=\operatorname{Hom}_{\Pi}(Y, X)=0$.

Proof. Let $\langle-,-\rangle$ be the Euler form on $K_{0}(\mathrm{fd} \widehat{\Pi})$. Since the extensions $E$ of $X, Y$ are layers by assumption, we have $\langle[X]+[Y],[X]+[Y]\rangle=\langle[E],[E]\rangle=2$. Thus

$$
2\langle[X],[Y]\rangle=\langle[X]+[Y],[X]+[Y]\rangle-\langle[X],[X]\rangle-\langle[Y],[Y]\rangle=-2 .
$$

Since $\operatorname{Ext}_{\widehat{\Pi}}^{1}(X, Y)=\operatorname{Ext}_{\Pi}^{1}(X, Y)$ is one-dimensional by assumption, it follows that $\operatorname{Hom}_{\Pi}(X, Y)=\operatorname{Hom}_{\widehat{\Pi}}(X, Y)=0$ and $\operatorname{Hom}_{\Pi}(Y, X)=D \operatorname{Ext}_{\widehat{\Pi}}^{2}(X, Y)=0$.

The following lemma is an analogue for doubletons of Lemma 3.3 .

Lemma 5.3. If $X, Y$ is a doubleton contained in $\mathrm{T}(w)$, and $\operatorname{Hom}_{\Pi}\left(X, S_{i}\right) \neq 0$ and $X \not S_{i}$, then $\ell\left(s_{i} w\right)=\ell(w)+1$ and $F_{i}(X), F_{i}(Y)$ form a doubleton contained in $\mathrm{T}\left(s_{i} w\right)$.

Proof. Since $\operatorname{Hom}_{\Pi}\left(X, S_{i}\right) \neq 0$, we have (Proposition $3.2($ a) $)$ that $\operatorname{Hom}_{\Pi}\left(S_{i}, X\right)=$ 0 . Since $\operatorname{Hom}_{\Pi}(X, Y)=0$ by Lemma [5.2 then $\operatorname{Hom}_{\Pi}\left(S_{i}, Y\right)=0$ also. Thus, by Proposition $3.2(\mathrm{~b}), F_{i}(X)$ and $F_{i}(Y)$ are layer modules. Since $F_{i}$ is an autoequivalence of $\mathrm{D}^{\mathrm{b}}(\mathrm{fd} \widehat{\Pi})$, it follows that $F_{i}(X), F_{i}(Y)$ still form a doubleton.

$F_{i}(X)$ belongs to $\mathrm{T}\left(s_{i} w\right)$ by Lemma $3.3(\mathrm{~b})$. If $\operatorname{Hom}_{\Pi}\left(Y, S_{i}\right) \neq 0$, then Lemma 3.3 is also directly applicable to $Y$, and tells us that $F_{i}(Y)$ also belongs to $\mathrm{T}\left(s_{i} w\right)$. In fact, the proof of Lemma 3.3 (b) is actually applicable to $Y$ even if $\operatorname{Hom}_{\Pi}\left(Y, S_{i}\right)=0$ - all that is really needed is that $\operatorname{Hom}_{\Pi}\left(S_{i}, Y\right)=0$, and we have this. Thus, $F_{i}(Y)$ also belongs to $\mathrm{T}\left(s_{i} w\right)$. 
The following lemma is an analogue for doubletons of Lemma 3.4 .

Lemma 5.4. If $X, Y$ is a doubleton, then there exists $v \in W$ such that $F(v)(X)$ and $F(v)(Y)$ are simple modules.

Proof. Suppose that at least one of $X$ and $Y$ is not simple. Without loss of generality, suppose that $X$ is not simple. Choose $S_{i}$ so that $\operatorname{Hom}_{\Pi}\left(X, S_{i}\right) \neq 0$. Applying Lemma [5.3. we see that $F_{i}(X)$ and $F_{i}(Y)$ are a doubleton in Fac $I_{s_{i}}$. Assume that one of $F_{i}(X)$ and $F_{i}(Y)$ is not simple. Repeat the previous procedure. As in the proof of Lemma 3.4 the procedure must terminate, at which point we have obtained a doubleton of simple modules.

Proof of Proposition 5.1. Suppose that we have a doubleton $A, C$, with $B$ the extension of $C$ by $A$. We will establish that there exists a hexagon in weak order such that one side of it is labelled $(A, B, C)$. By Lemma [5.4 there exists $v \in W$ such that $F(v)(A), F(v)(C)$ form a doubleton of simple modules, say $S_{i}, S_{j}$. As in the proof of Theorem 1.2, we conclude that $A, C$ are isomorphic to $D\left(I(v) / I\left(s_{i} v\right)\right.$ ) and $D\left(I(v) / I\left(s_{j} v\right)\right)$. By Lemma 3.5, these are the labels of the Hasse arrows $w_{0} v^{-1} \rightarrow w_{0} v^{-1} s_{i}$ and $w_{0} v^{-1} \rightarrow w_{0} v^{-1} s_{j}$ which form the two top arrows of the desired hexagon. Since the extension groups between $A$ and $C$ are one-dimensional, the side of the hexagon whose arrows are labelled $(A, ?, C)$, has $B$ as the label of its middle side.

It is immediate from the definition that if $A \geq B$ in the doubleton extension order, then $A$ is a subfactor of $B$. The converse does not hold in general. In Section 6, we define conventions regarding modules over the preprojective algebra of type $D_{n}$. In the notation of that section, in type $D_{4}$,

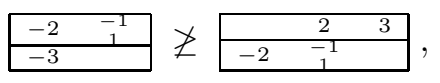

even though the first of these modules is a subfactor of the second.

We now show that the converse does hold in type $A_{n}$. We denote by $\mathscr{S}$ the set of non-revisiting walks on the double quiver $\bar{Q}$. By definition, these are walks in $Q$ which follow a sequence of arrows either with or against the direction of the arrow and which do not visit any vertex more than once. We identify a walk and its reverse walk.

Let $I_{\text {cyc }}$ denote the ideal of $\Pi$ generated by all 2 -cycles and let $\bar{\Pi}:=\Pi / I_{\text {cyc }}$. To any $p \in \mathscr{S}$, we can associate an indecomposable $\bar{\Pi}$-module $X_{p}$ called a string module, and these exhaust the indecomposable $\bar{\Pi}$-modules, see [WW].

Theorem 5.5. Suppose $\Pi$ is the preprojective algebra of type $A_{n}$.

(a) The layer modules for $\Pi$ are exactly the indecomposable $\bar{\Pi}$-modules (which are exactly the string modules).

(b) The doubleton extension order on layer modules is the opposite of subfactor order.

Proof. (a) Note that $I_{\text {cyc }}$ is generated as an ideal of $\Pi$ by the sum of all the twocycles, which is a central element $x \in \Pi$. If $X$ is a $\Pi$-module, then multiplication by $x$ is a surjective morphism onto $I_{\text {cyc }} X \subset X$. Therefore, if $X$ is a stone, $I_{\text {cyc }} X$ must be zero. Conversely, it is easy to see that any string module for $\bar{\Pi}$ is a stone.

(b) As mentioned above, we need only show that if $A$ is a subfactor of $B$, then $A \geq B$. If $A$ is a submodule or a quotient module of $B$, then $B$ is part of a doubleton having $A$ as one of its two extensions; if not, then $A$ is a submodule of some layer module $C$ which is a quotient of $B$, and we apply the same argument twice. 
Example 5.6. Let $n=3$. Then the Hasse quiver of $\mathscr{S}$ with respect to the opposite of subfactor order is the following:

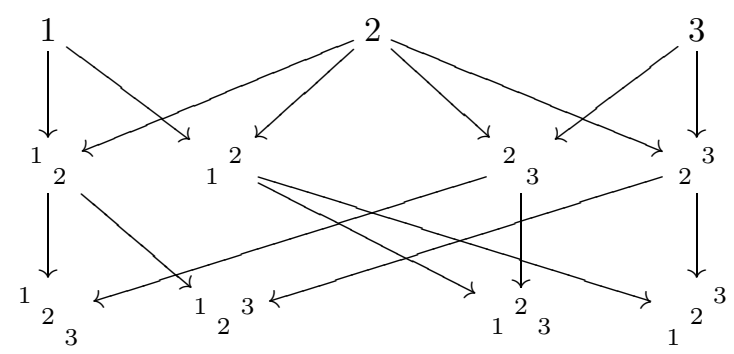

In light of Theorems 1.4 and 5.5, this quiver is the Hasse quiver of the forcing order on join-irreducible elements in type $A_{3}$. Compare [R1, Figure 4].

\section{Combinatorial Description of indecomposable $\tau$-Rigid Modules}

Let $\Pi$ be a preprojective algebra of Dynkin type and $W$ the corresponding Weyl group. A combinatorial description of join-irreducible elements in $W$ is well-known for type $A$ and $D$. We refer to Sections $1.5,2.1,2.4$, and 2.6 in $[\mathrm{BB}$. for type A, and Section 8.2 in $\mathrm{BB}$ for type D. (The description of join-irreducible elements is not given explicitly in $\mathrm{BB}$, but it is easily worked out from the combinatorial models developed there.) On the other hand, recall from Theorem4.1 that we have a bijection

$$
J: \mathrm{j}-\operatorname{Irr} W \rightarrow \mathrm{i} \tau^{-} \text {-rigid } \Pi
$$

given by $J(w):=(\Pi / I(w)) e_{i}$ for a unique arrow $w \rightarrow w s_{i}$ in the Hasse quiver of $W$ starting at $w$.

The main result of this section is to give a combinatorial description of the $\Pi$ module $J(w)$ for each join-irreducible element $w \in W$ in type $A$ or $D$. See Theorem 6.1 for type $A$ and Theorems 6.5 and 6.12 for type $D$. It will be interesting to compare our results with Bongartz's description of bricks for type $A$ and $D$ [Bon.

6.1. Type $A$. Let $\Pi$ be a preprojective algebra of type $A_{n}$. It is given by a quiver

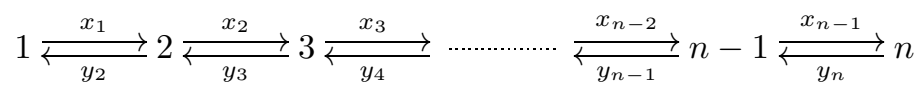

with relations $x_{1} y_{2}=0, x_{i} y_{i+1}=y_{i} x_{i-1}$ for $2 \leq i \leq n-1$ and $y_{n} x_{n-1}=0$. We denote by $S_{\ell}$ the simple $\Pi$-module corresponding to the vertex $\ell$, and by $P_{\ell}$ the projective cover of $S_{\ell}$.

Let $W=\mathfrak{S}_{n+1}$ be the Weyl group of $\Pi$. We use the convention that the product $w w^{\prime}$ of elements $w, w^{\prime} \in W$ is given by $\left(w w^{\prime}\right)(i)=w\left(w^{\prime}(i)\right)$ for $i \in\{1, \ldots, n+1\}$. The elements of $W$ are the permutations

$$
w=\left[i_{1}, \ldots, i_{n+1}\right] .
$$

This is join-irreducible if and only if there exists $\ell \in\{1, \ldots, n\}$ such that

$$
i_{1}<\cdots<i_{\ell}>i_{\ell+1}<\cdots<i_{n+1} .
$$

In this case, we say that $w$ is of type $\ell$. There exists a unique arrow $w \rightarrow w s_{\ell}$ starting at $w$ in the Hasse quiver of $W$. The number of join-irreducible elements of type $\ell$ is given by

$$
\left(\begin{array}{c}
n+1 \\
\ell
\end{array}\right)-1
$$

and therefore $\#$ j-Irr $W=2^{n+1}-n-2$. 
As a quiver representation, $P_{\ell}$ is given by

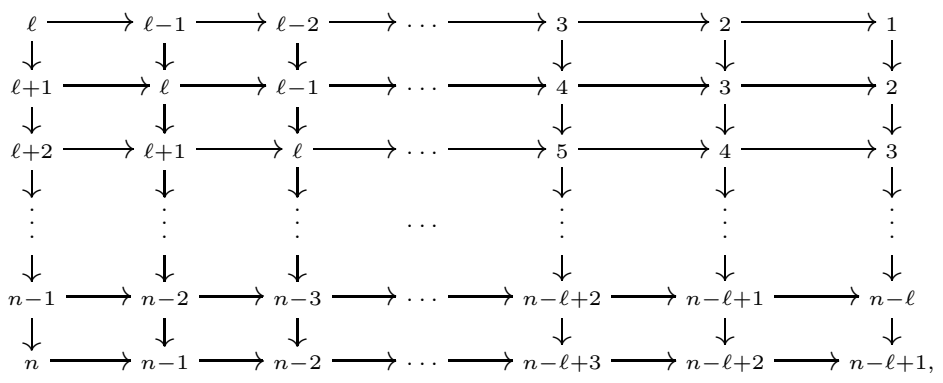

where each number $i$ shows a $k$-vector space $k$ lying on the vertex $i$, and each arrow is the identity map of $k$. Submodules (respectively, factor modules) of $P_{\ell}$ correspond bijectively to subquivers that are closed under successors (respectively, predecessors).

We represent $P_{\ell}$ in abbreviated form as an array of numbers in rows as follows:

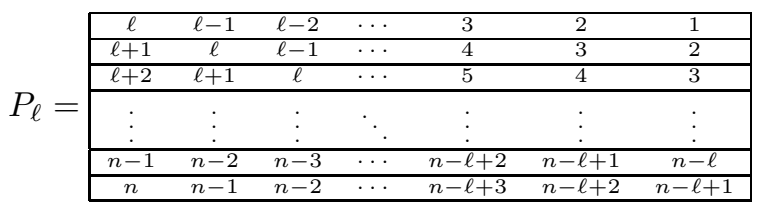

Submodules and factor modules are similarly represented by sub-arrays of $P_{\ell}$, as, for example, in the following theorem.

Theorem 6.1. Let $w=\left[i_{1}, \ldots, i_{n+1}\right] \in W$ be a join-irreducible element of type $\ell$. Then $J(w)$ is a factor module of $P_{\ell}$ which has the form

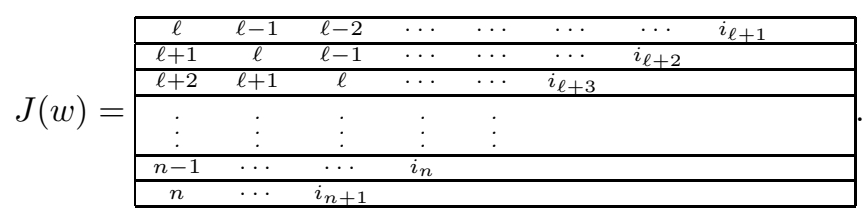

In particular, any factor module of $P_{\ell}$ is indecomposable $\tau^{-}$-rigid.

Note that $i_{m} \leq m$ holds for any $m \in\{\ell+1, \ldots, n+1\}$, and if $i_{m}=m$ holds, then the row starting at $m-1$ is empty. For example, for $n=5$ and $w=$ [351246],

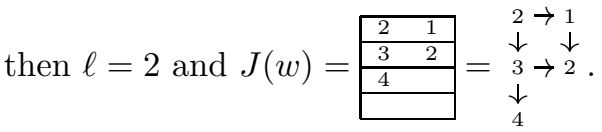

To prove this, we need the following easy observation.

Lemma 6.2. Let $w=\left[i_{1}, \ldots, i_{n+1}\right] \in W$ be a join-irreducible element of type $\ell$. Then we have a reduced expression $w=x_{n+1} x_{n} \cdots x_{\ell+2} x_{\ell+1}$, where $x_{m}=$ $s_{i_{m}} s_{i_{m}+1} \cdots s_{m-2} s_{m-1}$ for $m \in\{\ell+1, \ldots, n+1\}$.

Proof. If $\ell=n$, then $w=[1,2, \cdots, i-1, i+1, \ldots, n+1, i]$ holds for some $i \in$ $\{1, \ldots, n\}$. Thus $w=x_{n+1}$ holds clearly. In the rest, assume $\ell \neq n$, and let $v:=x_{n+1}^{-1} w$. Since $x_{n+1}^{-1}$ is a cyclic permutation $\left(n+1, n, \ldots, i_{n+1}+1, i_{n+1}\right)$, it peserves the total order on $\left\{i_{1}, \ldots, i_{n}\right\}$, and therefore $v$ is a join-irreducible element of type $\ell$ and clearly satisfies $v(n+1)=n+1$. Since $x_{n+1}^{-1}$ fixes any element in $\left\{i_{\ell+1}, \ldots, i_{n}\right\}$, we have $v(m)=i_{m}$ for any $m \in\{\ell+1, \ldots, n\}$ and $v(n+1)=n+1$. Inductively on $n$, we have $v=x_{n} \cdots x_{\ell+1}$, and therefore $w=x_{n+1} x_{n} \cdots x_{\ell+1}$.

Now we are ready to prove Theorem 6.1. 
Proof of Theorem 6.1. Clearly we have

$$
I\left(x_{\ell+1}\right) e_{\ell}=I_{i_{\ell+1}} \cdots I_{\ell} e_{\ell}=\begin{array}{|ccccccccc|}
\hline & & & i_{\ell+1}+1 & \cdots & 1 \\
\hline \ell+1 & \ell & \cdots & \cdots & \cdots & \cdots & 2 \\
\hline \ell+2 & \ell+1 & \cdots & \cdots & \cdots & \cdots & 3 \\
\hline \vdots & \vdots & \vdots & \vdots & \vdots & \vdots & \vdots \\
\hline n & n-1 & \cdots & \cdots & \cdots & \cdots & n-\ell+1 \\
\hline
\end{array}
$$

and

$$
I\left(x_{\ell+2} x_{\ell+1}\right) e_{\ell}=\begin{array}{|ccccccc|}
\hline \multicolumn{10}{|c}{} & i_{\ell+1}+1 & \cdots & 1 \\
\cline { 2 - 8 } & & & i_{\ell+2}+1 & \cdots & \cdots & 2 \\
\hline \ell+2 & \ell+1 & \cdots & \cdots & \cdots & \cdots & 3 \\
\hline \vdots & \vdots & \vdots & \vdots & \vdots & \vdots & \vdots \\
\hline n & n-1 & \cdots & \cdots & \cdots & \cdots & n-\ell+1 \\
\hline
\end{array}
$$

Repeating similar calculations, we have

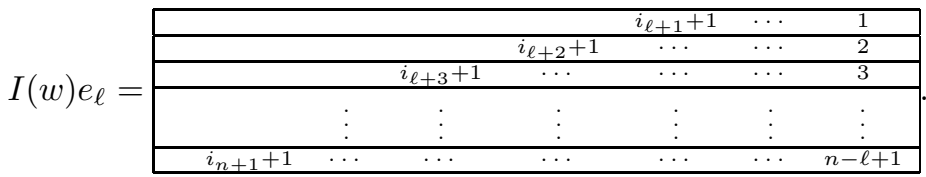

Therefore $J(w)=(\Pi / I(w)) e_{\ell}$ has the desired form.

Example 6.3. Let $w=[n-\ell+2, n-\ell+3, \ldots, n+1,1,2, \ldots, n-\ell+1]$ be a join-irreducible element of type $\ell$. Then we have a reduced expression

$$
w=x_{n+1} x_{n} \cdots x_{\ell+2} x_{\ell+1} \text { for } x_{m}=s_{m-\ell} \cdots s_{m-2} s_{m-1},
$$

and the corresponding indecomposable $\tau^{-}$-rigid $\Pi$-module is $J(w)=P_{\ell}$.

Example 6.4. Consider type $A_{3}$.

We have the following 3 join-irreducible elements of type 1 .

$J(2134)=J\left(s_{1}\right)=\square, \quad J(3124)=J\left(s_{2} \cdot s_{1}\right)=\frac{1}{2}, \quad J(4123)=J\left(s_{3} \cdot s_{2} \cdot s_{1}\right)=$\begin{tabular}{l}
$\frac{1}{2}$ \\
\hline 3
\end{tabular}.

We have the following 5 join-irreducible elements of type 2 .

$J(1324)=J\left(s_{2}\right)=2, \quad J(2314)=J\left(s_{1} s_{2}\right)=\begin{array}{ll}2 & 1\end{array}, \quad J(1423)=J\left(s_{3} \cdot s_{2}\right)=\begin{aligned} & 2 \\ & 3\end{aligned}$ $J(2413)=J\left(s_{3} \cdot s_{1} s_{2}\right)=$\begin{tabular}{|ll}
2 & 1 \\
3 &
\end{tabular},$\quad J(3412)=J\left(s_{2} s_{3} \cdot s_{1} s_{2}\right)=$\begin{tabular}{|ll}
2 & 1 \\
\hline 3 & 2 \\
\hline
\end{tabular} .

We have the following 3 join-irreducible elements of type 3 . $J(1243)=J\left(s_{3}\right)=3, \quad J(1342)=J\left(s_{2} s_{3}\right)=\begin{array}{ll}3 & 2\end{array}$, $J(2341)=J\left(s_{1} s_{2} s_{3}\right)=\begin{array}{lll}3 & 2 & 1\end{array}$.

6.2. Type $D$. Let $\Pi$ be a preprojective algebra of type $D_{n}$. Then $\Pi$ is given by a quiver

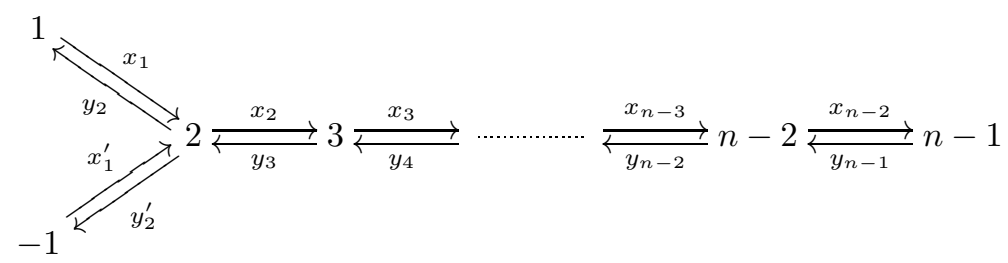

with relations $x_{1} y_{2}=0, x_{1}^{\prime} y_{2}^{\prime}=0, x_{2} y_{3}=y_{2} x_{1}+y_{2}^{\prime} x_{1}^{\prime}, x_{i} y_{i+1}=y_{i} x_{i-1}$ for $3 \leq i \leq n-2$ and $y_{n-1} x_{n-2}=0$. Let $S_{\ell}$ be the simple $\Pi$-module corresponding to the vertex $\ell$, and let $P_{\ell}$ be the projective cover of $S_{\ell}$.

The Weyl group $W$ of $\Pi$ is the group of automorphisms $w$ of the set $\{ \pm 1, \ldots, \pm n\}$ satisfying $w(-\ell)=-w(\ell)$ for any $\ell$ and $\#\{\ell \in\{1, \ldots, n\} \mid w(\ell)<0\}$ is even. Setting $i_{\ell}=w(\ell)$ for $\ell \in\{1, \ldots, n\}$, we write $w \in W$ as a sequence

$$
w=\left[i_{1}, \ldots, i_{n}\right] \in\{ \pm 1, \ldots, \pm n\}^{n}
$$

satisfying the following two conditions.

- $\left|i_{1}\right|, \ldots,\left|i_{n}\right|$ is a permutation of $1, \ldots, n$.

- The number of negative integers is even. 
We often denote $-i$ by $\underline{i}$. The simple reflections in $W$ are given by

$$
s_{-1}=[\underline{2}, \underline{1}, 3,4, \ldots, n] \text { and } s_{\ell}=[1, \ldots, \ell-1, \ell+1, \ell, \ell+2, \ldots, n]
$$

with $\ell \in\{1, \ldots, n-1\}$. The length of $w=\left[i_{1}, \ldots, i_{n}\right]$ is given by

$$
\ell(w)=\#\left\{1 \leq \ell<m \leq n \mid i_{\ell}>i_{m}\right\}+\#\left\{1 \leq \ell<m \leq n \mid-i_{\ell}>i_{m}\right\} .
$$

For $\ell \in\{1, \ldots, n-1\}, \ell\left(w s_{\ell}\right)-\ell(w)$ is 1 if $i_{\ell}<i_{\ell+1}$, and -1 otherwise. Moreover $\ell\left(w s_{-1}\right)-\ell(w)$ is 1 if $-i_{1}<i_{2}$, and -1 otherwise. Therefore an element $w=$ $\left[i_{1}, \ldots, i_{n}\right] \in W$ is join-irreducible if and only if one of the following conditions is satisfied.

- $i_{1}<\cdots<i_{n}$ and $-i_{1}>i_{2}$.

- There exists $\ell \in\{1, \ldots, n-1\}$ such that $i_{1}<\cdots<i_{\ell}>i_{\ell+1}<\cdots<i_{n}$ and $-i_{1}<i_{2}$.

We say that $w$ is of type -1 (respectively, type $\ell$ ) if the first (respectively, second) condition is satisfied. Then there exists a unique arrow $w \rightarrow w s_{\ell}$ starting at $w$ in the Hasse quiver of $W$. The number of join-irreducible elements of type $\ell \neq \pm 1$ (respectively, $1,-1$ ) is

$$
2^{n-\ell}\left(\begin{array}{l}
n \\
\ell
\end{array}\right)-1 \quad\left(\text { respectively, } 2^{n-1}-1,2^{n-1}-1\right)
$$

and therefore $\#$ j-Irr $W=3^{n}-n 2^{n-1}-n-1$.

As a quiver representation, $P_{\ell}$ for $\ell= \pm 1$ is given by

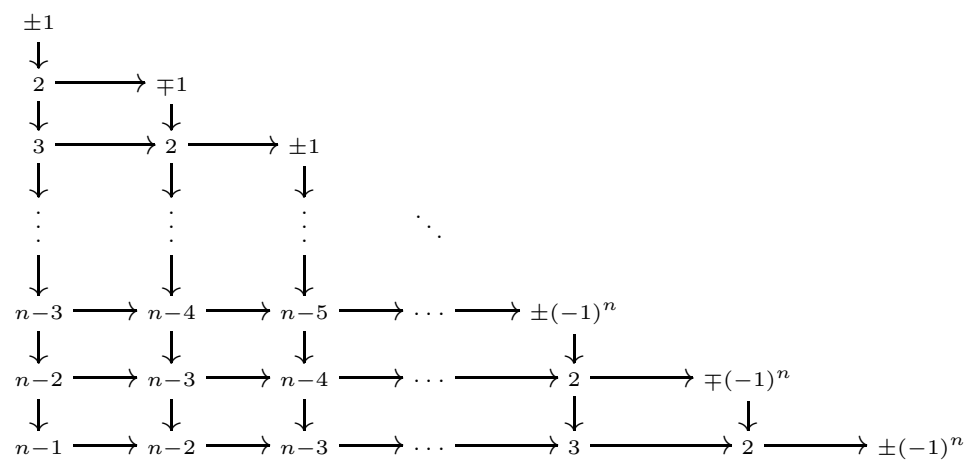

where each number $i$ shows a $k$-vector space $k$ lying on the vertex $i$, and each arrow is the identity map of $k$. Again, submodules (respectively, factor modules) of $P_{\ell}$ correspond bijectively to subquivers that are closed under successors (respectively, predecessors).

We represent $P_{\ell}$ in abbreviated form as an array of numbers in rows as follows:

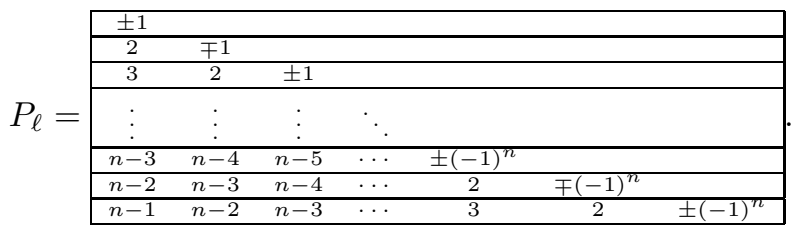

Submodules and factor modules of $P_{\ell}$ are again represented by subarrays.

Theorem 6.5. Let $w=\left[i_{1}, \ldots, i_{n}\right] \in W$ be a join-irreducible element of type $\ell= \pm 1$. Then $J(w)$ is a factor module of $P_{\ell}$ which has the form

$$
J(w)=\begin{array}{|ccccc|}
\hline i_{2}^{\prime} & & & & \\
\hline 2 & i_{3}^{\prime} & & & \\
\hline 3 & \cdots & i_{4}^{\prime} & & \\
\hline \vdots & \vdots & \vdots & \ddots & \\
\hline n-2 & n-3 & \cdots & \cdots & i_{n-1}^{\prime} \\
\hline n-1 & n-2 & \cdots & i_{n}^{\prime} & \\
\hline
\end{array}
$$


where $i_{m}^{\prime}:=\max \left\{i_{m},(-1)^{m} \ell\right\}$ for $m \in\{2, \ldots, n\}$.

Note that $i_{m}^{\prime} \leq m$ holds for any $m \in\{2, \ldots, n\}$, and if $i_{m}^{\prime}=m$ holds, then the row starting at $m-1$ is empty. For example, for $n=6$ and $w=[5 \underline{31246}$, then $\ell=1$ and $J(w)=$\begin{tabular}{|ll}
1 & $\begin{array}{ll}1 \\
\downarrow \\
2\end{array}$ \\
2 & $\rightarrow-1$ \\
$\downarrow$ & $\downarrow$ \\
3 & $\rightarrow 2$ \\
$\downarrow$ & \\
4
\end{tabular}.

To prove this, we need the following observation.

Lemma 6.6. Let $w=\left[i_{1}, \ldots, i_{n}\right] \in W$ be a join-irreducible element of type $\ell= \pm 1$. Then we have a reduced expression $w=x_{n} x_{n-1} \cdots x_{3} x_{2}$, where

$$
x_{m}=\left\{\begin{array}{ll}
s_{i_{m}} s_{i_{m}+1} \cdots s_{m-2} s_{m-1} & \text { if } i_{m} \geq 2 \\
s_{(-1)^{m} \ell} s_{2} \cdots s_{m-2} s_{m-1} & \text { if } i_{m}<2
\end{array} \quad \text { for } m \in\{2, \ldots, n\} .\right.
$$

Proof. The case $n=2$ can be checked directly. In the rest, we assume $n \geq 3$. Assume that the assertion holds for $n-1$. Again, let $v:=x_{n}^{-1} w$.

Assume $i_{n}>0$. Then $v$ is obtained from $w$ by replacing $i_{n}$ with $n$ and then, for each $i_{j}$ with $i_{n}<\left|i_{j}\right|$ replacing $i_{j}$ by an entry with the same sign but absolute value $\left|i_{j}\right|-1$. In particular, $v$ is a join-irreducible element of type $\ell$ and satisfies $v(n)=n$. Fix $m \in\{2, \ldots, n-1\}$. If $i_{m}>0$, then $v(m)=i_{m}$ holds, and if $i_{m}<0$, then $v(m)<0$ holds. By our assumption on induction, we have $v=x_{n-1} \cdots x_{2}$, and therefore $w=x_{n} x_{n-1} \cdots x_{2}$.

Assume $i_{n}<0$. In this case, we have $w=\left[(-1)^{n-1} n, \underline{n-1}, \underline{n-2}, \ldots, \underline{1}\right]$ and $\ell=$ $(-1)^{n-1}$. Thus $x_{n}^{-1}=[\underline{n}, \underline{1}, 2, \ldots, n-1]$ and $v=\left[(-1)^{n-1}(n-1), \underline{n}-2, \ldots, \underline{2}, 1, n\right]$ hold. Therefore $v$ is a join-irreducible element of type $\ell$ and satisfies $v(n)=n$. Since $v(m)<2$ holds for any $m \in\{2, \ldots, n-1\}$, we have $v=x_{n-1} \cdots x_{2}$ by our assumption on induction, and therefore $w=x_{n} x_{n-1} \cdots x_{2}$.

Now we are ready to prove Theorem 6.5.

Proof of Theorem 6.5. Using Lemma 6.6, one can calculate $I(w)$ as in the proof of Theorem 6.1, and we obtain the desired assertion.

Example 6.7. Let $w=\left[n, \underline{n-1}, \underline{n-2}, \ldots, \underline{2},(-1)^{n}\right]$ be a join-irreducible element of type 1 . Then we have a reduced expression

$$
w=x_{n} x_{n-1} \cdots x_{3} x_{2} \text { for } x_{m}=s_{(-1)^{m}} s_{2} s_{3} \cdots s_{m-1},
$$

and the corresponding indecomposable $\tau^{-}$-rigid $\Pi$-module is $J(w)=P_{1}$.

Similarly, let $w=\left[\underline{n}, \underline{n-1}, \underline{n-2}, \ldots, \underline{2},(-1)^{n+1}\right]$ be a join-irreducible element of type -1 . Then we have a reduced expression

$$
w=x_{n} x_{n-1} \cdots x_{3} x_{2} \text { for } x_{m}=s_{(-1)^{m+1}} s_{2} s_{3} \cdots s_{m-1},
$$

and the corresponding indecomposable $\tau^{-}$-rigid $\Pi$-module is $J(w)=P_{-1}$.

Example 6.8. Consider type $D_{4}$.

We have the following 7 join-irreducible elements of type 1 .

$$
\begin{aligned}
& J(2134)=J\left(s_{1}\right)=1, \quad J(3124)=J\left(s_{2} \cdot s_{1}\right)=\frac{1}{2}, \\
& J(3 \underline{21} 4)=J\left(s_{-1} s_{2} \cdot s_{1}\right)=\begin{array}{|ll}
\frac{1}{2} & -1
\end{array}, \quad J(4123)=J\left(s_{3} \cdot s_{2} \cdot s_{1}\right)=\begin{array}{l}
\frac{1}{2} \\
\hline 3
\end{array},
\end{aligned}
$$

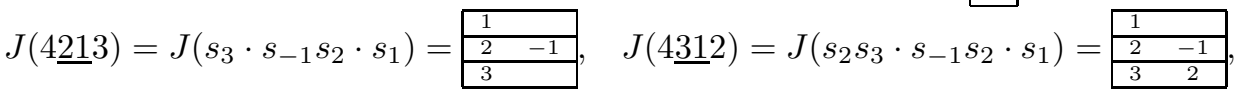

$$
\begin{aligned}
& J(4 \underline{32} 1)=J\left(s_{1} s_{2} s_{3} \cdot s_{-1} s_{2} \cdot s_{1}\right)=\begin{array}{|lll|}
\hline \begin{array}{lll}
1 & & \\
\hline 2 & -1 & \\
\hline 3 & 2 & 1 \\
\hline
\end{array}
\end{array}
\end{aligned}
$$

We have the following 7 join-irreducible elements of type -1 . 


$$
\begin{aligned}
& J(\underline{21} 34)=J\left(s_{-1}\right)=\square-1, \quad J(\underline{31} 24)=J\left(s_{2} \cdot s_{-1}\right)=\frac{-1}{2}, \\
& J(\underline{32} 14)=J\left(s_{1} s_{2} \cdot s_{-1}\right)=\begin{array}{|ll}
\frac{-1}{2} & 1
\end{array}, \quad J(\underline{41} 23)=J\left(s_{3} \cdot s_{2} \cdot s_{-1}\right)=\frac{-1}{2},
\end{aligned}
$$

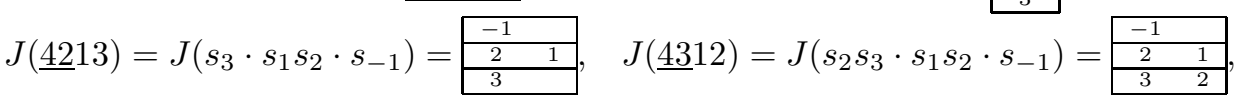

$$
\begin{aligned}
& J(\underline{4321})=J\left(s_{-1} s_{2} s_{3} \cdot s_{1} s_{2} \cdot s_{-1}\right)=\begin{array}{|lll|}
\hline-1 & & \\
\hline 2 & 1 & \\
\hline 3 & 2 & -1 \\
\hline
\end{array} .
\end{aligned}
$$

In the rest of this section, let $w$ be a join-irreducible element $i_{1}<\cdots<i_{\ell}>$ $i_{\ell+1}<\cdots<i_{n}$ of type $\ell \neq \pm 1$. Note that all integers in $i_{2}, \ldots, i_{\ell}$ must be positive, and therefore $w$ can be recovered from the latter part $i_{\ell+1}, \ldots, i_{n}$.

We need the following preparation on the structure of $P_{\ell}$.

Lemma 6.9. Let $\alpha$ and $\beta$ be scalars satisfying $\alpha+\beta=1$. As a quiver representation, $P_{\ell}$ with $\ell \neq \pm 1$ is given by

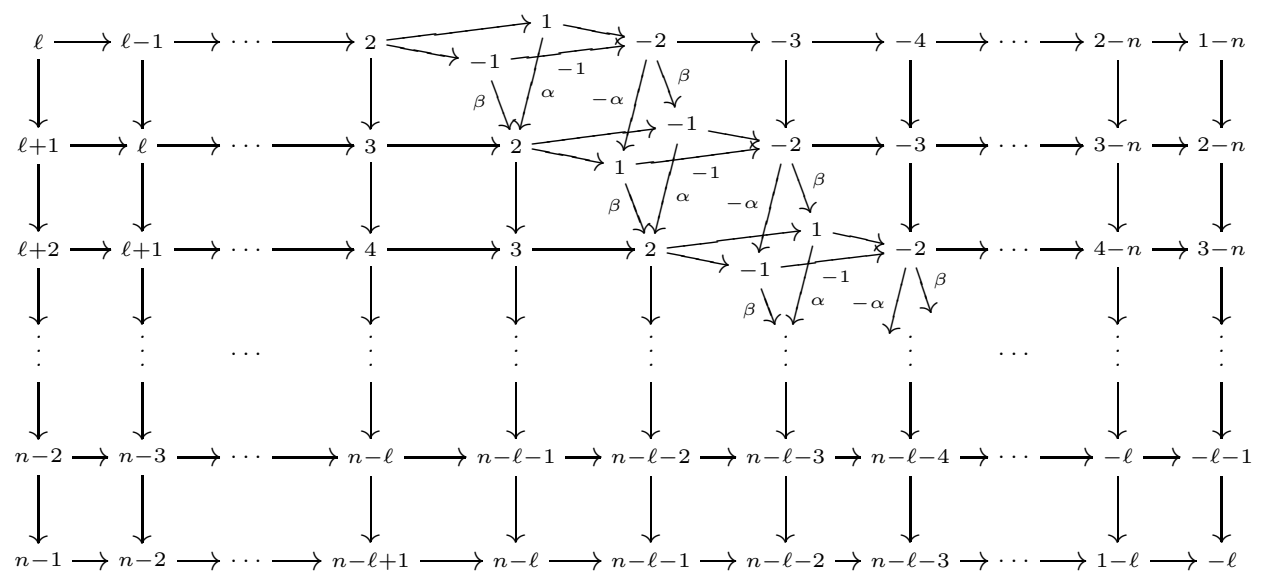

$(6.1)$

where each number $i$ shows a $k$-vector space $k$ lying on the vertex $i$ if $i \geq-1$ and $-i$ if $i \leq-2$. Each unlabelled arrow is the identity map of $k$, and each arrow labelled by a scalar $\gamma$ is a linear map multiplying by $\gamma$.

Proof. Since all relations of $\Pi$ are satisfied, this gives a $\Pi$-module $X$. It is easy to check that $X$ is generated by $\ell$ in the upper left corner. Thus we have a surjective morphism $\pi: P_{\ell} \rightarrow X$ of $\Pi$-modules. On the other hand, it is well-known that the Loewy length of any idecomposable projective $\Pi$-module is equal to $h-1$, where $h$ is the Coxeter number. For type $D_{n}$, we have $h-1=2 n-3$. Since the length of the path from $\ell$ in the left corner to $-\ell$ in the lower right corner is $2 n-4$, the above $\pi$ must be an isomorphism.

Example 6.10. Let $n=6$. Then $P_{2}$ is given by the following quiver representations, where the left one is the case $(\alpha, \beta)=(0,1)$, and the right one is the case $(\alpha, \beta)=(1,0)$.
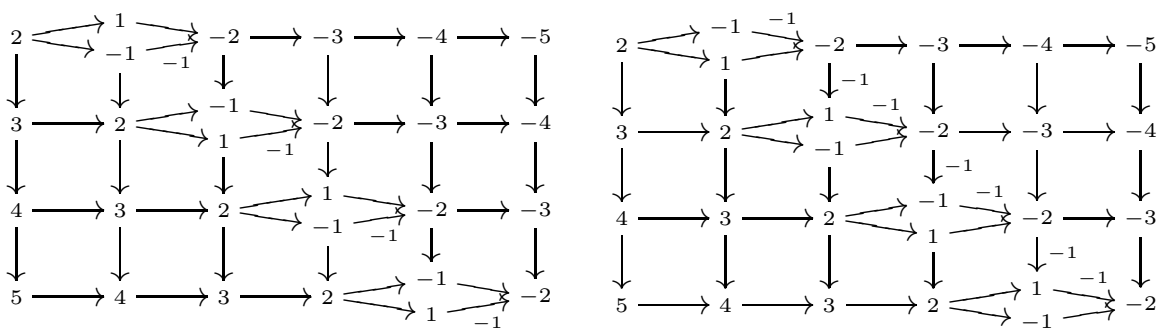
We write the quiver $P_{\ell}$ of (6.1) in abbreviated form as the following array of numbers.

\begin{tabular}{|ccccccccccccc|}
\hline$\ell$ & $\ell-1$ & $\cdots$ & 2 & -1 & -2 & $\cdots$ & $1-\ell$ & $-\ell$ & $-\ell-1$ & $\cdots$ & $2-n$ & $1-n$ \\
\hline$\ell+1$ & $\ell$ & $\cdots$ & 3 & 2 & -1 & $\cdots$ & $2-\ell$ & $1-\ell$ & $-\ell$ & $\cdots$ & $3-n$ & $2-n$ \\
\hline$\vdots$ & $\vdots$ & $\ddots$ & $\ddots$ & $\ddots$ & $\ddots$ & $\ddots$ & $\ddots$ & $\ddots$ & $\ddots$ & $\ddots$ & $\vdots$ & $\vdots$ \\
\hline$n-2$ & $n-3$ & $\cdots$ & $\ell$ & $\ell-1$ & $\ell-2$ & $\cdots$ & -1 & -2 & -3 & $\cdots$ & $-\ell$ & $-\ell-1$ \\
\hline$n-1$ & $n-2$ & $\cdots$ & $\ell+1$ & $\ell$ & $\ell-1$ & $\cdots$ & 2 & -1 & -2 & $\cdots$ & $1-\ell$ & $-\ell$ \\
\hline
\end{tabular}

The description of factor $P_{ \pm 1}$ was no more complicated than the analogous description in type $A$. However, for $\ell>1$, the description of factor modules of $P_{\ell}$ is much more complicated in type $D$. For example, consider the direct sum $k^{2}$ of $k$ 's corresponding to -2 in the first row and 2 in the second row. Then subspaces of $k^{2}$ generate distinct submodules of $P_{\ell}$. Fortunately, to describe $J(w)$ for $w$ join-irreducible, we only need the following special class of factor modules.

Definition 6.11. Let $S$ be a subarray of the array 6.2. We say that $S$ is predecessor-closed if it is closed under predecessors in the quiver (6.1).

Now we fix scalars $\alpha$ and $\beta$ satisfying $\alpha+\beta=1$. We say that $S$ is $(\alpha, \beta)$ predecessor-closed if it is closed under predecessors in the subquiver of (6.1) obtained by removing all arrows indexed by the scalar 0 . An $(\alpha, \beta)$-predecessorclosed subarray $S$ gives a factor module of $P_{\ell}$ in a natural way. Clearly, if $(\alpha, \beta) \neq$ $(1,0),(0,1)$, then $S$ is $(\alpha, \beta)$-predecessor-closed if and only if it is predecessor-closed.

For $m \in\{2, \ldots, n-1\}$, let $C(m, j)$ be the following subset of numbers in the row of (6.2) starting at $m$.

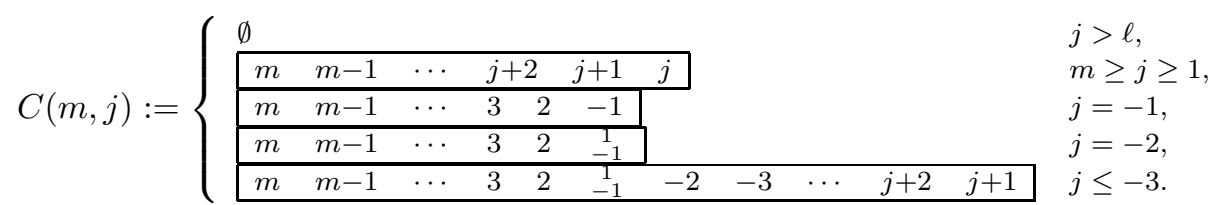

We simply write $C(m, j)$ as $\begin{array}{lllll}m & m-1 & m-2 & \cdots & j^{\prime}\end{array}$, where $j^{\prime}:=j$ if $j \geq-1$ and $j^{\prime}:=j+1$ if $j \leq-2$.

Let $S(w)$ be the subarray of the array (6.2) given by

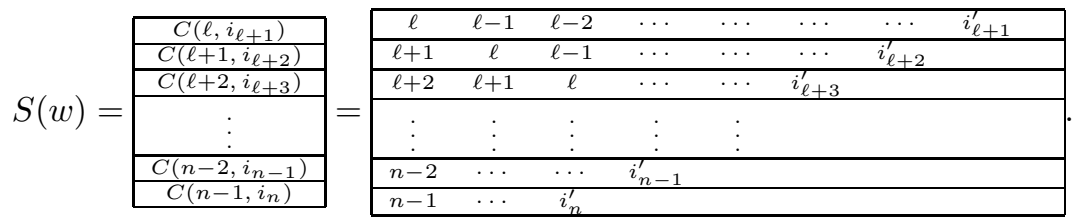

Note that $i_{m}^{\prime} \leq m$ holds for any $m \in\{\ell+1, \ldots, n\}$, and if $i_{m}^{\prime}=m$ holds, then the row starting at $m-1$ is empty.

Theorem 6.12. Let $w=\left[i_{1}, \ldots, i_{n}\right] \in W$ be a join-irreducible element of type $\ell \neq \pm 1$, and $S(w)$ subarray of the array (6.2) described in (6.3).

(a) If $\{1,2\} \not \subset\left\{\left|i_{\ell+1}\right|, \ldots,\left|i_{n}\right|\right\}$, then $S(w)$ is predecessor-closed.

(b) If $\{1,2\} \subset\left\{\left|i_{\ell+1}\right|, \ldots,\left|i_{n}\right|\right\}$, then $S(w)$ is $(1,0)$ or $(0,1)$-predecessor-closed.

In either case, we have a factor module of $P_{\ell}$ corresponding to $S(w)$.

(c) $J(w)$ is the factor module of $P_{\ell}$ corresponding to $S(w)$.

For example, let $n=6$ and $w=[\underline{3} 4 \underline{5} 126]$ and $w^{\prime}=[34 \underline{52} 16]$. Then

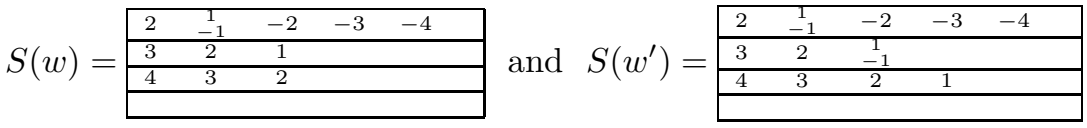


Thus $S(w)$ is $(0,1)$-predecessor-closed and $S\left(w^{\prime}\right)$ is $(1,0)$-predecessor-closed, and

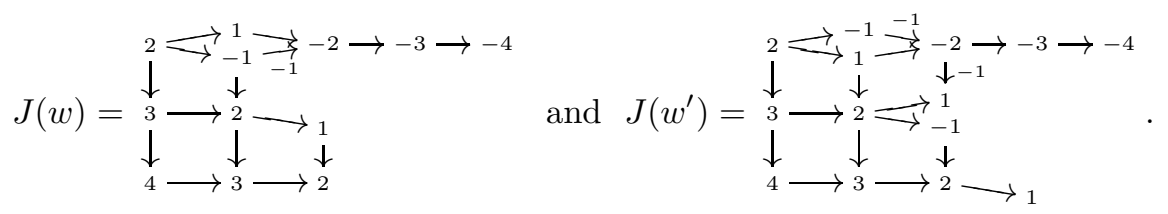

To prove Theorem 6.12 we need some preparation. Let $w=\left[i_{1}, \ldots, i_{n}\right] \in W$ be a join-irreducible element of type $\ell$. For each $m \in\{\ell+1, \ldots, n\}$, let

$$
j_{m}:=i_{m}+\#\left\{m^{\prime}\left|m \leq m^{\prime} \leq n,\right| i_{m^{\prime}}|\leq| i_{m} \mid\right\}
$$

Clearly $i_{m}>0$ implies $j_{m}=i_{m}+1>1$, and $i_{m}<0$ implies $j_{m} \leq 0$. In the latter case, we have $j_{m}=-\#\left\{m^{\prime}\left|1 \leq m^{\prime} \leq \ell,\right| i_{m^{\prime}}|\leq| i_{m} \mid\right\}$. Therefore $j_{\ell+1} \leq j_{\ell+2} \leq$ $\cdots \leq j_{n}$ holds.

Lemma 6.13. Let $w=\left[i_{1}, \ldots, i_{n}\right] \in W$ be a join-irreducible element of type $\ell \neq$ \pm 1 . Then we have a reduced expression $w=x_{n} x_{n-1} \cdots x_{\ell+2} x_{\ell+1}$, where $x_{m}$ for $m \in\{\ell+1, \ldots, n\}$ is given by

$$
x_{m}= \begin{cases}s_{i_{m}} s_{i_{m}+1} \cdots s_{m-1} & \text { if } j_{m}>0, \\ s_{\epsilon_{m}} s_{2} s_{3} \cdots s_{m-1} \text { for } \epsilon_{m}:=(-1)^{\#\left\{m^{\prime} \mid m \leq m^{\prime} \leq n, i_{m^{\prime}}<0\right\}} & \text { if } j_{m}=0, \\ s_{-1} s_{1} s_{2} s_{3} \cdots s_{m-1} & \text { if } j_{m}=-1, \\ s_{-j_{m}} s_{-j_{m}-1} \cdots s_{3} s_{2} s_{-1} s_{1} s_{2} s_{3} \cdots s_{m-1} & \text { if } j_{m}<-1 .\end{cases}
$$

Proof. Since $i_{1}<i_{2}$ and $-i_{1}<i_{2}$, we have $0<i_{2}<\cdots<i_{\ell}$. Let $h_{1}<\cdots<h_{\ell+1}$ be the reordering of

- $i_{1}, i_{2}, \ldots, i_{\ell}, i_{\ell+1}$ if $j_{\ell+1}>0$ or $\left(j_{\ell+1}=0\right.$ and $\left.i_{1}>0\right)$,

- $-i_{1}, i_{2}, \ldots, i_{\ell},-i_{\ell+1}$ if $j_{\ell+1}<0$ or $\left(j_{\ell+1}=0\right.$ and $\left.i_{1}<0\right)$,

and let $v:=\left[h_{1}, \ldots, h_{\ell+1}, i_{\ell+2}, \ldots, i_{n}\right] \in W$. It is easy to check that $v$ is either an identity or join-irreducible of type $\ell+1$. We will show

$$
w x_{\ell+1}^{-1}=v \text { and } \ell(w)-\ell\left(x_{\ell+1}\right)=\ell(v) .
$$

Then the assertion follows inductively.

Let $t$ be the unique integer satisfying $h_{t}=\left|i_{\ell+1}\right|$.

Assume $j_{\ell+1}>0$ and so $i_{\ell+1}>0$. Since all positive integers smaller than $i_{\ell+1}$ appear in $\left|i_{1}\right|, \ldots,\left|i_{\ell}\right|$ we have $t=i_{\ell+1}$. Thus (6.5) follows from $x_{\ell+1}=s_{t} s_{t+1} \cdots s_{\ell}$.

Assume $j_{\ell+1}=0$ and so $i_{\ell+1}<0$. Since all positive integers smaller than $i_{\ell+1}$ appear in $\left|i_{\ell+2}\right|, \ldots,\left|i_{n}\right|$, we have $t=1$. If $i_{1}>0$, then $\epsilon_{\ell+1}=1$ holds, and (6.5) follows from $x_{\ell+1}=s_{1} s_{2} s_{3} \cdots s_{\ell}$. If $i_{1}<0$, then $\epsilon_{\ell+1}=-1$ holds, and (6.5) follows from $x_{\ell+1}=s_{-1} s_{2} s_{3} \cdots s_{\ell}$.

Assume $j_{\ell+1}<0$ and so $i_{\ell+1}<0$. Then $t=1-j_{\ell+1}$ holds, and (6.5) follows easily from $x_{\ell+1}=s_{t-1} s_{t-2} \cdots s_{3} s_{2} s_{-1} s_{1} s_{2} s_{3} \cdots s_{\ell}$.

Now we are ready to prove Theorem 6.12

Proof of Theorem 6.12, (a) and (b) are easily checked.

(c) Choose scalars $\alpha$ and $\beta$ such that $S(w)$ is $(\alpha, \beta)$-predecessor-closed. Using the quiver representation of $P_{\ell}$ given in (6.1) and the reduced expression of $w$ given in Lemma 6.13, we calculate $I(w) e_{\ell}$. Dividing into three cases, we show that the first row of $J(w)$ coincides with that of $S(w)$.

Assume $j_{\ell+1}>0$. By (6.4), the first row of $I\left(x_{\ell+1}\right) e_{\ell}$ is given by

$$
\left\{\begin{array}{rrrrrrrrr|}
\hline i_{\ell+1}-1 & i_{\ell+1}-2 & \cdots & 2 & -1 & -2 & \cdots & 2-n & 1-n \\
& & & -1 & -2 & -3 & \cdots & 2-n & 1-n \\
& & & & & & & &
\end{array}\right.
$$


Since $0<i_{\ell+1}<i_{\ell+2}<\cdots<i_{n}$ holds, both $s_{i_{\ell+1}-1}$ (when $i_{\ell+1} \geq 2$ ) and $s_{-1}$ (when $i_{\ell+1}=1$ ) do not appear in $x_{n} x_{n-1} \cdots x_{\ell+2}$ by (6.4). Thus the first row of $I(w) e_{\ell}$ coincides with that of $I\left(x_{\ell+1}\right) e_{\ell}$, and the first row of $J(w)$ coincides with that of $S(w)$.

Assume $j_{\ell+1}<0$. By (6.4), the first row of $I\left(x_{\ell+1}\right) e_{\ell}$ is given by

$$
\begin{array}{|llllll}
\hline j_{\ell+1}-1 & j_{\ell+1}-2 & j_{\ell+1}-3 & \cdots & 2-n & 1-n \\
\hline
\end{array}
$$

Let $m_{0}:=\min \left\{m|\ell+1 \leq m \leq n,| i_{m}|\leq| i_{\ell+1} \mid\right\}$, which equals $j_{\ell+1}-i_{\ell+1}+\ell$. We show that, for any $\ell+1 \leq m \leq m_{0}$, the first row of $I\left(x_{m} x_{m-1} \cdots x_{\ell+1}\right) e_{\ell}$ is

$$
\begin{array}{|lllll}
\hline j_{\ell+1}-m+\ell & j_{\ell+1}-m+\ell-1 & \cdots & 2-n & 1-n \\
\hline
\end{array}
$$

The case $m=\ell+1$ was shown above. Assume that this is the case for $m-1\left(<m_{0}\right)$. It suffices to show that $s_{-j_{\ell+1}+m-1-\ell}$ appears in $x_{m}$, and that $s_{-j_{\ell+1}+m-\ell}$ does not appear in the left side of $s_{-j_{\ell+1}+m-1-\ell}$ in $x_{m}$. Since $-j_{\ell+1} \leq \ell$ holds, we have

$$
2 \leq-j_{\ell+1}+m-1-\ell \leq m-1 \text {. }
$$

If $j_{m} \geq 0$, then the assertion follows from (6.4) and

$$
i_{m} \leq i_{m_{0}}-m_{0}+m \leq-i_{\ell+1}-m_{0}+m-1=-j_{\ell+1}+m-1-\ell .
$$

If $j_{m}<0$, then the assertion follows from (6.4) and $-j_{m} \leq-j_{\ell+1}<-j_{\ell+1}+m-1-\ell$. Inductively, we have shown that the first row of $I\left(x_{m_{0}} x_{m_{0}-1} \cdots x_{\ell+1}\right) e_{\ell}$ is

$$
\begin{array}{|lllll|}
\hline i_{\ell+1} & i_{\ell+1}-1 & \cdots & 2-n & 1-n \\
\hline
\end{array}
$$

since $j_{\ell+1}-m_{0}+\ell=i_{\ell+1}$. Since $0<-i_{\ell+1}<i_{m_{0}+1}<\cdots<i_{n}$ holds, $s_{-i_{\ell+1}}$ does not appear in $x_{n} x_{n-1} \cdots x_{m_{0}+1}$. Thus the first row of $I(w) e_{\ell}$ coincides with (6.6), and the first row of $J(w)$ coincides with that of $S(w)$.

Assume $j_{\ell+1}=0$. By (6.4), the first row of $I\left(x_{\ell+1}\right) e_{\ell}$ is given by

\begin{tabular}{|llllll|}
\hline$-\epsilon_{\ell+1}$ & -2 & -3 & $\cdots$ & $2-n$ & $1-n$ \\
\hline
\end{tabular}

By a similar argument as in the case $j_{\ell+1}<0$, one can check that the first row of $J(w)$ coincides with that of $S(w)$.

We have completed to show that the first row of $J(w)$ coincides with that of $S(w)$. All rows of $I\left(x_{\ell+1}\right) e_{\ell}$ except the first one coincide with those of $P_{\ell}$ since $s_{\ell+1}$ does not appear in $x_{\ell+1}$. Repeating the same calculation, all rows of $J(w)$ coincide with that of $S(w)$.

Example 6.14. Let $w=\left[(-1)^{n-\ell}, 2, \ldots, \ell, \underline{n}, \underline{n-1}, \ldots, \underline{\ell+1}\right]$ be a join-irreducible element of type $\ell$. Then we have a reduced expression

$$
w=x_{n} x_{n-1} \cdots x_{\ell+2} x_{\ell+1} \text { for } x_{m}=s_{\ell} \cdots s_{3} s_{2} s_{-1} s_{1} s_{2} s_{3} \cdots s_{m-1},
$$

and the corresponding indecomposable $\tau^{-}$-rigid $\Pi$-module is $J(w)=P_{\ell}$.

Example 6.15. Consider type $D_{4}$.

We have the following 7 join-irreducible elements of type 3 .

$J(1243)=J\left(s_{3}\right)=3, \quad J(1342)=J\left(s_{2} s_{3}\right)=\begin{array}{ll}3 & 2\end{array}$,

$J(2341)=J\left(s_{1} s_{2} s_{3}\right)=$\begin{tabular}{lll}
\hline 3 & 2 & 1
\end{tabular},$\quad J(\underline{2} 34 \underline{1})=J\left(s_{-1} s_{2} s_{3}\right)=\begin{array}{lll}3 & 2 & -1\end{array}$,

$J(\underline{1} 34 \underline{2})=J\left(s_{-1} s_{1} s_{2} s_{3}\right)=$\begin{tabular}{|lll}
\hline 3 & 2 & -1
\end{tabular},$\quad J(\underline{1} 24 \underline{3})=J\left(s_{2} s_{-1} s_{1} s_{2} s_{3}\right)=$\begin{tabular}{lllll}
\hline 3 & 2 & 1 & -2
\end{tabular} ,

$J(\underline{1} 23 \underline{4})=J\left(s_{3} s_{2} s_{-1} s_{1} s_{2} s_{3}\right)=$\begin{tabular}{lllll}
\hline 3 & 2 & 1 & -2 & -2
\end{tabular}.

We have the following 23 join-irreducible elements of type 2 . They are predecessorclosed unless otherwise specified.

$$
\begin{aligned}
& J(1324)=J\left(s_{2}\right)=2, \quad J(\underline{2} 3 \underline{1} 4)=J\left(s_{-1} s_{2}\right)=\frac{2 \quad-1}{2}, \\
& J(2314)=J\left(s_{1} s_{2}\right)=\begin{array}{ll}
2 & 1
\end{array}, \quad J(1423)=J\left(s_{3} \cdot s_{2}\right)=\frac{2}{3} \text {, } \\
& J(2413)=J\left(s_{3} \cdot s_{1} s_{2}\right)=\begin{array}{|ll}
\hline 2 & 1 \\
\hline 3 &
\end{array}, \quad J(\underline{2} 4 \underline{1} 3)=J\left(s_{3} \cdot s_{-1} s_{2}\right)=\begin{array}{|ll}
\hline 2 & -1 \\
\hline 3 &
\end{array}, \\
& J(\underline{1} 3 \underline{2} 4)=J\left(s_{-1} s_{1} s_{2}\right)=\begin{array}{|ll}
\hline 2 & -1
\end{array}, \quad J(\underline{1} 4 \underline{2} 3)=J\left(s_{3} \cdot s_{-1} s_{1} s_{2}\right)=\begin{array}{|ll|}
\hline 2 & -1 \\
\hline 3 &
\end{array}
\end{aligned}
$$




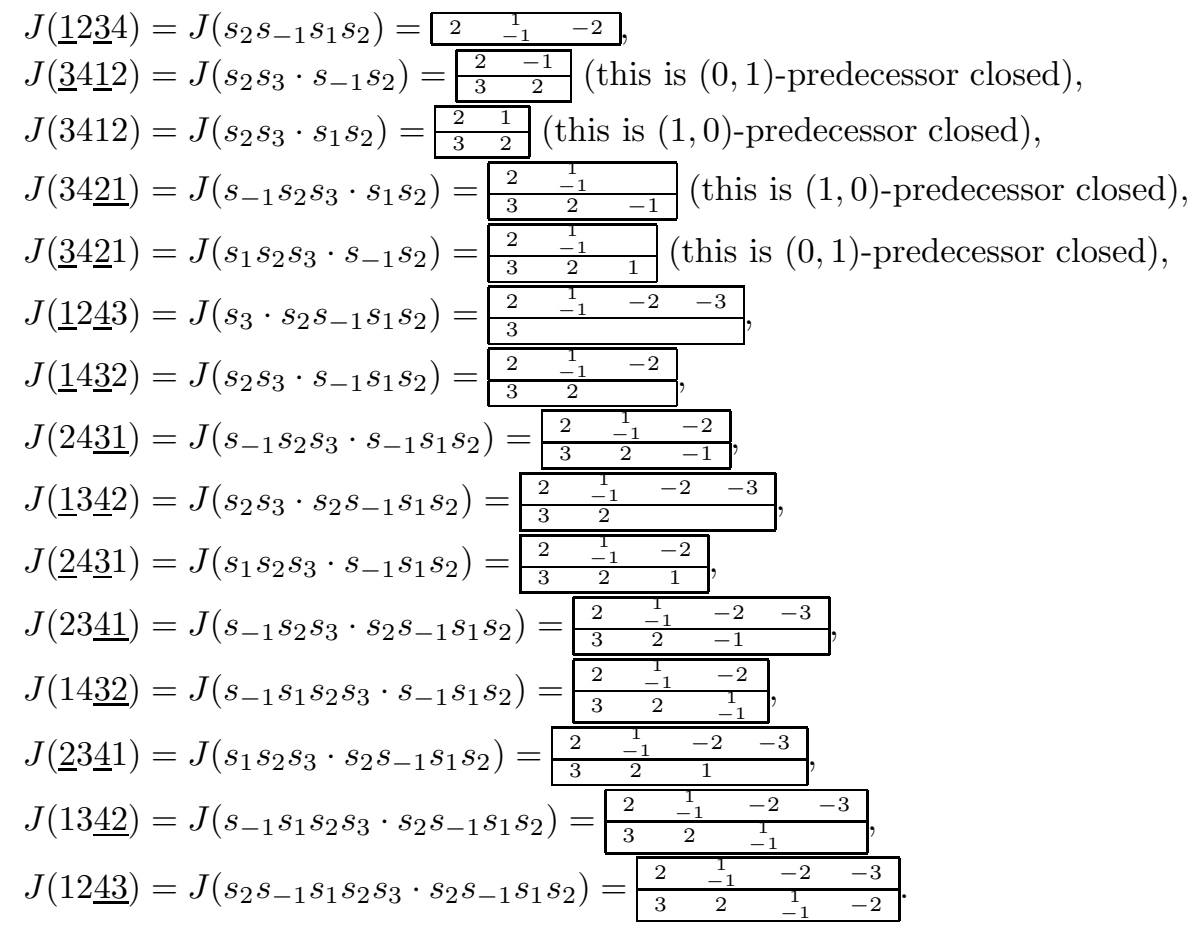

\section{REFERENCES}

[AIR] T. Adachi, O. Iyama, I. Reiten, $\tau$-tilting theory, Compos. Math. 150 (2014), no. 3, 415-452.

$[\mathrm{AM}]$ T. Aihara, Y. Mizuno, Classifying tilting complexes over preprojective algebras of Dynkin type, arXiv:1509.07387.

[A] C. Amiot, Cluster categories for algebras of global dimension 2 and quivers with potential, Ann. Inst. Fourier (Grenoble) 59 (2009), no. 6, 2525-2590.

[AIRT] C. Amiot, O. Iyama, I. Reiten, G. Todorov, Preprojective algebras and c-sortable words, Proc. Lond. Math. Soc. (3) 104 (2012), no. 3, 513-539.

[AS] M. Auslander, S. O. Smalo, Almost split sequences in subcategories, J. Algebra 69 (1981), no. $2,426-454$.

[BKT] P. Baumann, J. Kamnitzer, P. Tingley, Affine Mirković-Vilonen polytopes, Publ. Math. Inst. Hautes Études Sci. 120 (2014), 113-205.

[BGL] D. Baer, W. Geigle, H. Lenzing, The preprojective algebra of a tame hereditary Artin algebra, Comm. Algebra 15 (1987), no. 1-2, 425-457.

[Bol] B. Bolten, Spiegelungsfunktoren für präprojektive Algebren, Diplomarbeit (2010), Bonn.

[Bon] J. Bongartz, Endotrivial modules over preprojective algebras, Master's Thesis (2015), Bonn.

[BIRS] A. Buan, O. Iyama, I. Reiten, J. Scott, Cluster structures for 2-Calabi-Yau categories and unipotent groups, Compos. Math. 145 (2009), no. 4, 1035-1079.

[BB] A. Björner and F. Brenti, Combinatorics of Coxeter groups, Graduate Texts in Mathematics, 231. Springer, New York, 2005.

[CLM] N. Caspard, C. Le Conte de Poly-Barbut and M. Morvan, Cayley lattices of finite Coxeter groups are bounded, Adv. in Appl. Math. 33 (2004), no. 1, 71-94.

[CH] W. Crawley-Boevey, M. P. Holland, Noncommutative deformations of Kleinian singularities, Duke Math. J. 92 (1998), no. 3, 605-635.

[D] A. Day, Congruence normality: the characterization of the doubling class of convex sets, Algebra Universalis 31 (1994), no. 3, 397-406.

[DIJ] L. Demonet, O. Iyama, G. Jasso, $\tau$-tilting finite algebras, g-vectors and brick- $\tau$-rigid correspondence, arXiv: 1503.00285

[DIRRT] L. Demonet, O. Iyama, I. Reiten, N. Reading, H. Thomas, Lattices of torsion classes and their algebraic quotients, in preparation.

[DR] V. Dlab, C. M. Ringel, The preprojective algebra of a modulated graph, Representation theory, II (Proc. Second Internat. Conf., Carleton Univ., Ottawa, Ont., 1979), pp. 216-231, Lecture Notes in Math., 832, Springer, Berlin-New York, 1980.

[GLS] C. Geiss, B. Leclerc, J. Schröer, Kac-Moody groups and cluster algebras, Adv. Math. 228 (2011), no. 1, 329-433. 
[HHKU] D. Happel, S. Hartlieb, O. Kerner, L. Unger, On perpendicular categories of stones over quiver algebras, Comment. Math. Helv. 71 (1996), no. 3, 463-474.

[IR] O. Iyama, I. Reiten, Fomin-Zelevinsky mutation and tilting modules over Calabi-Yau algebras, Amer. J. Math. 130 (2008), no. 4, 1087-1149.

[IRTT] O. Iyama, I. Reiten, H. Thomas, G. Todorov, Lattice structure of torsion classes for path algebras, Bull. Lond. Math. Soc. 47 (2015), no. 4, 639-650.

[KS] M. Kashiwara, Y. Saito, Geometric construction of crystal bases, Duke Math. J. 89 (1997), no. 1, 9-36.

[KL] O. Kerner, F. Lukas, Regular stones of wild hereditary algebras, J. Pure Appl. Algebra 93 (1994), no. 1, 15-31.

[K] Y. Kimura, Tilting theory of preprojective algebras and c-sortable elements, arXiv:1405.4087.

[Le] B. Leclerc, Cluster structures on strata of flag varieties, to appear in Adv. Math., arXiv:1402.4435

[Lu] G. Lusztig, Quivers, perverse sheaves, and quantized enveloping algebras, J. Amer. Math. Soc. 4 (1991), no. 2, 365-421.

[Ma] F. Marks, Homological embeddings for preprojective algebras, arXiv:1512.02442

[Mi] Y. Mizuno, Classifying $\tau$-tilting modules over preprojective algebras of Dynkin type, Math. Z. 277 (2014) no. 3-4, 665-690.

[N] H. Nakajima, Instantons on ALE spaces, quiver varieties, and Kac-Moody algebras, Duke Math. J. 76 (1994), no. 2, 365-416.

[ORT] S. Oppermann, I. Reiten, H. Thomas, Quotient closed subcategories of quiver representations, Compos. Math. 151 (2015), no. 3, 568-602.

[R1] N. Reading, Lattice congruences of the weak order. Order 21 (2004) no. 4, 315-344.

[R2] N. Reading, Lattice Theory of the Poset of Regions, in Lattice Theory: Special Topics and Applications, ed. G. Grätzer and F. Wehrung, Birkhäuser, to appear.

[R3] N. Reading, Finite Coxeter Groups and the Weak Order, in Lattice Theory: Special Topics and Applications, ed. G. Grätzer and F. Wehrung, Birkhäuser, to appear.

[Ri] C. M. Ringel, Representations of K-species and bimodules, J. Algebra 41 (1976), no. 2, 269302.

[ST] P. Seidel, R. Thomas, Braid group actions on derived categories of coherent sheaves, Duke Math. J. 108 (2001), no. 1, 37-108.

[SY1] Y. Sekiya, K. Yamaura, Tilting theoretical approach to moduli spaces over preprojective algebras, Algebr. Represent. Theory 16 (2013), no. 6, 1733-1786.

[SY2] Y. Sekiya, K. Yamaura, private communications.

[WW] B. Wald, J. Waschbüsch, Tame biserial algebras, J. Algebra 95 (1985), no. 2, 480-500.

O. Iyama: Graduate School of Mathematics, Nagoya University, Chikusa-ku, Nagoya, 464-8602 JAPAN

E-mail address: iyama@math.nagoya-u.ac.jp

$U R L:$ http://www.math.nagoya-u.ac.jp/ iyama/

N. Reading: Department of Mathematics, North Carolina State University, Raleigh, NC 27695-8205, USA

E-mail address: reading@math.ncsu.edu

URL: http://www4.ncsu.edu/ nreadin/

I. Reiten: Department of Mathematical Sciences Norges teknisk-Naturvitenskapelige UNIVERsiteT 7491 TrondheIM NorWAY

E-mail address: idun.reiten@math.ntnu.no

$U R L:$ http://www.ntnu.edu/employees/idun.reiten

H. Thomas: Département de mathématiques, Université du Québec à Montréal, CP 8888, Succursale Centre-Ville, Montréal, QC, H3C 3P8, Canada

E-mail address: hugh.ross.thomas@gmail.com

$U R L:$ http: //www.lacim.uqam.ca/ hugh 